Pacific

Journal of

Mathematics

\title{
SPHERICAL CR DEHN SURGERIES
}

Miguel Acosta 


\title{
SPHERICAL CR DEHN SURGERIES
}

\author{
Miguel Acosta
}

\begin{abstract}
Consider a three-dimensional cusped spherical CR manifold $M$ and suppose that the holonomy representation of $\pi_{1}(M)$ can be deformed in such a way that the peripheral holonomy is generated by a nonparabolic element. We prove that, in this case, there is a spherical CR structure on some Dehn surgeries of $M$. The result is very similar to R. Schwartz's spherical CR Dehn surgery theorem, but has weaker hypotheses and does not give the uniformizability of the structure. We apply our theorem in the case of the Deraux-Falbel structure on the figure eight knot complement and obtain spherical CR structures on all Dehn surgeries of slope $-3+r$, for $r \in \mathbb{Q}^{+}$ small enough.
\end{abstract}

\section{Introduction}

The celebrated theorem of hyperbolic Dehn surgeries of Thurston [2002] says that all but a finite number of Dehn surgeries of a one-cusped hyperbolic manifold $M$ admit complete hyperbolic structures with developing maps and holonomy representations close to those of $M$. The same question arises for other geometric structures. We focus here on spherical CR structures, i.e., structures modeled on the boundary at infinity of the complex hyperbolic plane with group of automorphisms $\mathrm{PU}(2,1)$. Schwartz [2007] shows a spherical CR Dehn surgery theorem that gives, under some convergence hypotheses, uniformizable spherical CR structures on some Dehn surgeries on a cusped spherical CR manifold. In this paper, we prove a similar theorem using techniques coming from $(G, X)$-structures and the geometry of $\partial_{\infty} \mathbb{H}_{\mathbb{C}}^{2}$ instead of the alternative approach of discreteness of group representations and actions on $\mathbb{U}_{\mathbb{C}}^{2}$. Theorem 3.23 has weaker hypotheses than Schwartz's theorem, but we obtain geometric structures on the surgeries for which we do not know whether they are uniformizable.

For the reader, the example to keep in mind, treated in Section 4, is the DerauxFalbel structure on the figure-eight knot complement constructed in [Deraux and Falbel 2015]. For this example, Deraux [2014] shows that there is a one-parameter family of spherical CR uniformizations on the figure-eight knot complement with

MSC2010: 32V05, 57M25, 57M50.

Keywords: spherical CR, Dehn surgery, $(G, X)$-structures, figure-eight knot. 
parabolic peripheral holonomy containing this structure. Falbel, Guilloux, Koseleff, Rouillier, and Thistlethwaite [Falbel et al. 2016] describe the $\mathrm{SL}_{3}(\mathbb{C})$-character variety of the fundamental group of the figure-eight knot complement. They give an explicit parametrization for the component in $\mathrm{SU}(2,1)$ containing the holonomy representation of the Deraux-Falbel structure. This component also gives rise to spherical CR structures near the Deraux-Falbel structure. With this parametrization and Theorem 3.23, we obtain the following theorem:

Theorem. Let $M$ be the figure-eight knot complement. For the usual ${ }^{1}$ marking of the peripheral torus of $M$ :

(1) There exist infinitely many spherical CR structures on the Dehn surgery of $M$ of slope -3 .

(2) There exists $\delta>0$ such that for all $r \in \mathbb{Q} \cap] 0, \delta$, there is a spherical CR structure on the Dehn surgery of $M$ of slope $-3+r$.

In Section 2, we recall some properties about $\mathbb{M}_{\mathbb{C}}^{2}, \partial_{\infty} \mathbb{H}_{\mathbb{C}}^{2}$, and $\mathrm{PU}(2,1)$ and set some notation. We look in detail at the dynamics of one-parameter subgroups of $\mathrm{PU}(2,1)$ acting on $\partial_{\infty} \mathbb{M}_{\mathbb{C}}^{2}$. Understanding these dynamics will be crucial in the proof of the surgery theorem. Section 3 deals with deformation of $(G, X)$ structures and fixes some notation and a marking of a peripheral torus in order to state the main theorem of this paper, Theorem 3.23. In Section 4, we apply Theorem 3.23 in the case of the Deraux-Falbel structure, by checking the hypotheses and looking at the deformation space as given in [Falbel et al. 2016]. Finally, in Section 5, we give a complete proof of the surgery theorem.

\section{Generalities on $\mathbb{U}_{\mathbb{C}}^{2}$ and its isometries}

In this section we recall some facts about the hyperbolic complex plane $\mathbb{U}_{\mathbb{C}}^{2}$ and its boundary at infinity $\partial_{\infty} \mathbb{U}_{\mathbb{C}}^{2}$ and set notation for them. We study the group of holomorphic isometries of $\mathbb{T}_{\mathbb{C}}^{2}$, identified with $\mathrm{PU}(2,1)$, by describing its oneparameter subgroups. Almost all stated results can be found in the thesis of Genzmer [2010] and in the book of Goldman [1999].

The space $\mathbb{H}_{\mathbb{C}}^{2}$ and its boundary at infinity. We begin by giving a construction of the hyperbolic complex plane. Let $V$ be a complex vector space of dimension 3 endowed with a Hermitian product $\langle\cdot, \cdot\rangle$. Denote by $\Phi$ the associated Hermitian form. We suppose that $\Phi$ has signature $(2,1)$. By setting

$$
\begin{aligned}
& V_{-}=\{v \in V-\{0\} \mid \Phi(v)<0\}, \\
& V_{0}=\{v \in V-\{0\} \mid \Phi(v)=0\}, \\
& V_{+}=\{v \in V-\{0\} \mid \Phi(v)>0\},
\end{aligned}
$$

\footnotetext{
${ }^{1}$ For us, the usual marking is the one given in [Thurston 2002].
} 
the complex hyperbolic plane is defined as $\mathbb{P} V_{-}$, endowed with the Hermitian metric induced by $\Phi$, and its boundary at infinity $\partial_{\infty} \mathbb{H}_{\mathbb{C}}^{2}$ is defined as $\mathbb{P} V_{0}$.

Notation 2.1. We will denote with usual parentheses "(" and ")" the objects before projectivization and with square brackets "[" and "]" the class of an object in the projectivized space.

From now on, we will use two different models of the complex hyperbolic plane, going from one to another by a conjugation. In both cases, the vector space is $V=\mathbb{C}^{3}$. For the details in the construction, see [Goldman 1999].

Notation 2.2. Let

$$
J_{1}=\left(\begin{array}{rrr}
1 & 0 & 0 \\
0 & 1 & 0 \\
0 & 0 & -1
\end{array}\right) \quad \text { and } \quad J_{2}=\left(\begin{array}{lll}
0 & 0 & 1 \\
0 & 1 & 0 \\
1 & 0 & 0
\end{array}\right) .
$$

They are the matrices of the Hermitian products $\langle\cdot, \cdot\rangle_{1}$ and $\langle\cdot, \cdot\rangle_{2}$ and they are conjugate by Cayley's matrix

$$
C=\frac{1}{\sqrt{2}}\left(\begin{array}{ccr}
1 & 0 & 1 \\
0 & \sqrt{2} & 0 \\
1 & 0 & -1
\end{array}\right)
$$

Definition 2.3. By identifying $V$ with $\mathbb{C}^{3}$ and $\langle\cdot, \cdot\rangle$ with $\langle\cdot, \cdot\rangle_{1}$, we obtain the ball model. We then have

$$
\mathbb{M}_{\mathbb{C}}^{2}=\left\{\left.\left[\begin{array}{c}
Z_{1} \\
Z_{2} \\
1
\end{array}\right] \in \mathbb{C P}^{2}|| Z_{1}\right|^{2}+\left|Z_{2}\right|^{2}<1\right\}
$$

and

$$
\partial_{\infty} \mathbb{H}_{\mathbb{C}}^{2}=\left\{\left.\left[\begin{array}{c}
Z_{1} \\
Z_{2} \\
1
\end{array}\right] \in \mathbb{C P}^{2}|| Z_{1}\right|^{2}+\left|Z_{2}\right|^{2}=1\right\} .
$$

With this model, we see that $\mathbb{U}_{\mathbb{C}}^{2}$ is homeomorphic to the ball $B^{4}$ and $\partial_{\infty} \mathbb{U}_{\mathbb{C}}^{2}$ is homeomorphic to the sphere $S^{3}$. The other model that we will consider is the Siegel model, more convenient for drawing pictures.

Definition 2.4. By identifying $V$ with $\mathbb{C}^{3}$ and $\langle\cdot, \cdot\rangle$ with $\langle\cdot, \cdot\rangle_{2}$, we obtain the Siegel model, with

$$
\partial_{\infty} \mathbb{U}_{\mathbb{C}}^{2}=\left\{\left[\begin{array}{c}
-\frac{1}{2}\left(|z|^{2}+i t\right) \\
z \\
1
\end{array}\right] \mid(z, t) \in \mathbb{C} \times \mathbb{R}\right\} \cup\left\{\left[\begin{array}{l}
1 \\
0 \\
0
\end{array}\right]\right\} .
$$


We can then identify $\partial_{\infty} \mathbb{H}_{\mathbb{C}}^{2}$ with $(\mathbb{C} \times \mathbb{R}) \cup\{\infty\}$. Removing the point at infinity, we obtain the Heisenberg group, defined as $\mathbb{C} \times \mathbb{R}$ with multiplication

$$
(w, s) *(z, t)=(w+z, s+t+2 \operatorname{Im}(w \bar{z})) .
$$

We are also going to use complex geodesics, which are intersections of complex lines of $\mathbb{P} V$ with $\mathbb{U}_{\mathbb{C}}^{2}$, and their boundaries at infinity, called $\mathbb{C}$-circles.

Holomorphic isometries of $\mathbb{Q}_{\mathbb{C}}^{2}$ and invariant flows. We defined above the complex hyperbolic space and have seen two of its models. The group of holomorphic isometries of this space is $\mathrm{PU}(2,1)$, as described below.

Notation 2.5. Let $\mathrm{U}(2,1)$ be the group of matrices of $\mathrm{GL}_{3}(\mathbb{C})$ such that $A^{*} J A=J$ for $J=J_{1}$ or $J_{2}$ (according to the model in which we work). Let $\operatorname{SU}(2,1)$ be the subgroup of matrices of determinant 1 and $\mathrm{PU}(2,1)$ its projectivization.

We state in detail a classification of the elements of $\mathrm{PU}(2,1)$. We use the notations and state the results of [Genzmer 2010, Chapter 1]. Isometries are classified by their fixed points in $\mathbb{Q}_{\mathbb{C}}^{2} \cup \partial_{\infty} \mathbb{M}_{\mathbb{C}}^{2}$.

An isometry $g \neq \mathrm{Id}$ of $\mathbb{H}_{\mathbb{C}}^{2}$ is called elliptic if it has at least one fixed point in $\mathbb{U}_{\mathbb{C}}^{2}$, parabolic if it is not elliptic and has exactly one fixed point in $\partial_{\infty} \mathbb{H}_{\mathbb{C}}^{2}$, and loxodromic if it is not elliptic and has exactly two fixed points in $\partial_{\infty} \mathbb{H}_{\mathbb{C}}^{2}$.

We can state this classification in terms of eigenvalues. The eigenvalues of an element of $\mathrm{PU}(2,1)$ are only defined up to multiplication by a cube root of 1 that we denote by $\omega$; we give a condition on the eigenvalues of a lift in $\operatorname{SU}(2,1)$.

Proposition 2.6. Let $U \in \mathrm{SU}(2,1)-\{\mathrm{Id}\}$. Then $U$ is in one of the three following cases:

(1) U has an eigenvalue $\lambda$ of modulus different from 1 . Then $[U]$ is loxodromic.

(2) $U$ has an eigenvector $v \in V_{-}$. Then $[U]$ is elliptic and its eigenvalues have modulus equal to 1 but are not all equal.

(3) All eigenvalues of $U$ have modulus 1 and $U$ has an eigenvector $v \in V_{0}$. Then $[U]$ is parabolic.

To refine this classification, we will consider different cases when there are double eigenvalues. We give the following definition:

Definition 2.7. Let $U \in \mathrm{SU}(2,1)-\{\mathrm{Id}\}$. We say that $U$ is regular if its three eigenvalues are different and unipotent if its three eigenvalues are equal (and so equal to a cube root of 1 ).

The definition extends to $\mathrm{PU}(2,1)$; we will speak of regular elements of $\mathrm{PU}(2,1)$. In that case the eigenvalues are well-defined up to multiplication by $\omega$. Thanks to the following remark, we know that regular elements are easier to manipulate. 
Remark 2.8. Let $[U] \in \mathrm{PU}(2,1)$ be a regular element. Then $[U]$ is determined by its three eigenvalues $\alpha, \beta, \gamma$ and its three fixed points $[u],[v],[w]$ in $\mathbb{C P}^{2}$.

It is possible to know if an element is regular only by knowing its trace, thanks to the following proposition.

Proposition 2.9 [Goldman 1999]. For $z \in \mathbb{C}$, let $f(z)=|z|^{4}-8 \operatorname{Re}\left(z^{3}\right)+18|z|^{2}-27$. Let $U \in \mathrm{SU}(2,1)$. Then $U$ is regular if and only if $f(\operatorname{tr}(U)) \neq 0$. Furthermore, if $f(\operatorname{tr}(U))<0$ then $[U]$ is regular elliptic, and if $f(\operatorname{tr}(U))>0$ then $[U]$ is loxodromic.

Remark 2.10. It is suitable to make two remarks about the proposition:

(1) For $\omega \in \mathbb{C}$ satisfying $\omega^{3}=1$, we have $f(z)=f(\omega z)$. Therefore, we can define the function $f \circ \operatorname{tr}$ on $\mathrm{PU}(2,1)$.

(2) For a parabolic element $[U]$, the equality $f(\operatorname{tr}(U))=0$ holds, but there are nonregular elliptic elements for which $f(\operatorname{tr}(U))=0$.

In order to study spherical CR structures and their surgeries, we will use the flows of vector fields associated to some elements of $\mathrm{PU}(2,1)$. The geometric objects that we are going to consider are invariant vector fields induced by elements of PU $(2,1)$. We begin by looking at an infinitesimal level: an element of the Lie algebra $\mathfrak{s u}(2,1)$ defines a vector field on $\partial_{\infty} \mathbb{H}_{\mathbb{C}}^{2}$ invariant under its exponential map.

Notation 2.11. Let $X \in \mathfrak{s u}(2,1)$. It defines a vector field on $\partial_{\infty} \mathbb{H}_{\mathbb{C}}^{2}$ invariant by $\exp (X)$ given at a point $x$ by

$$
\left.\frac{\mathrm{d}}{\mathrm{d} t}\right|_{t=0} \exp (t X) \cdot x
$$

Let $\phi_{t}^{X}$ be the flow of this vector field, so $\phi_{t}^{X}(x)=\exp (t X) \cdot x$. If there is no ambiguity for $X$, we will only write $\phi_{t}$.

Remark 2.12. If $[U] \in \mathrm{PU}(2,1)$ is close enough to a unipotent element, it defines a vector field on $\partial_{\infty} \mathbb{H}_{\mathbb{C}}^{2}$. Indeed, possibly after changing the lift, we can suppose that the eigenvalues of $U$ are near 1, and consider the vector field associated to $\log (U)$. Then, $\phi_{1}^{\log (U)}$ has the same action as $[U]$.

Description of isometries and invariant flows. We are going to describe briefly some elements of PU $(2,1)$, and classify each by its type and the dynamics of its action on $\mathbb{C P}^{2}$.

We are going to study the dynamics of some flows of the form $\phi_{t}^{\log (U)}$, where $U$ is close to a unipotent element. We describe here flows associated to regular elliptic, loxodromic and unipotent elements. 
Regular elliptic flows. Consider a regular elliptic element in $\mathrm{SU}(2,1)$, close to Id, in the ball model. Perhaps after a conjugation, we can suppose that it is equal to

$$
E_{\alpha, \beta, \gamma}=\left(\begin{array}{ccc}
e^{i \alpha} & 0 & 0 \\
0 & e^{i \beta} & 0 \\
0 & 0 & e^{i \gamma}
\end{array}\right)
$$

The flow of the associated vector field acts then on $\partial_{\infty} \mathbb{M}_{\mathbb{C}}^{2}$ by

$$
\phi_{t}^{\log \left(E_{\alpha, \beta, \gamma}\right)}\left[\begin{array}{c}
Z_{1} \\
Z_{2} \\
1
\end{array}\right]=\left[\begin{array}{c}
e^{i t(\alpha-\gamma)} Z_{1} \\
e^{i t(\beta-\gamma)} Z_{2} \\
1
\end{array}\right]=\left[\begin{array}{c}
e^{i t(2 \alpha+\beta)} Z_{1} \\
e^{i t(2 \beta+\alpha)} Z_{2} \\
1
\end{array}\right] .
$$

Remark 2.13. The flow stabilizes the two $\mathbb{C}$-circles

$$
C_{1}=\left[e_{1}\right]^{\perp} \cap \partial_{\infty} \mathbb{H}_{\mathbb{C}}^{2}=\left\{\left[\begin{array}{c}
0 \\
e^{i \theta} \\
1
\end{array}\right] \mid \theta \in \mathbb{R}\right\}
$$

and

$$
C_{2}=\left[e_{2}\right]^{\perp} \cap \partial_{\infty} \mathbb{H}_{\mathbb{C}}^{2}=\left\{\left[\begin{array}{c}
e^{i \theta} \\
0 \\
1
\end{array}\right] \mid \theta \in \mathbb{R}\right\},
$$

on which it acts as rotations by angles $2 \beta+\alpha$ and $2 \alpha+\beta$ respectively.

Remark 2.14. The centralizer of $E_{\alpha, \beta, \gamma}$ is

$$
C\left(E_{\alpha, \beta, \gamma}\right)=\left\{E_{\theta_{1}, \theta_{2},-\left(\theta_{1}+\theta_{2}\right)} \mid\left(\theta_{1}, \theta_{2}\right) \in \mathbb{R}^{2}\right\} .
$$

The orbits of this subgroup in $\partial_{\infty} \mathbb{H}_{\mathbb{C}}^{2}$ are $C_{1}, C_{2}$, and the subsets $T_{r}$ for $\left.r \in\right] 0,1$, defined by

$$
T_{r}=\left\{\left[\begin{array}{c}
Z_{1} \\
Z_{2} \\
1
\end{array}\right] \in \partial_{\infty} \mathbb{H}_{\mathbb{C}}^{2}|| Z_{2}|=r,| Z_{1} \mid=\sqrt{1-r^{2}}\right\} .
$$

The orbits $T_{r}$ are embedded tori in $\partial_{\infty} \mathbb{Q}_{\mathbb{C}}^{2}$ with core curves $C_{1}$ and $C_{2}$. They are all invariant under the action of $\phi_{t}^{\log \left(E_{\alpha, \beta, \gamma}\right)}$. We can see an example in Figure 1.

Have a look now at the orbits of the flow $\phi_{t}^{\log \left(E_{\alpha, \beta, \gamma}\right)}$. Notice that the orbit of a point is included in a unique torus $T_{r}$, and that every orbit included in $T_{r}$ is the image of a fixed orbit by an element $E_{\theta_{1}, \theta_{2},-\left(\theta_{1}+\theta_{2}\right)}$. Thus, the torus $T_{r}$ is foliated by these orbits. We fix $r \in] 0,1$ [, and consider two cases:

Case 1: $\alpha / \beta \notin \mathbb{Q}$. In this case, the angles of rotation in $T_{r}$ for $\phi_{t}$ are $(2 \alpha+\beta) t$ and $(2 \beta+\alpha) t$. Since their quotient is irrational, an orbit is an injective immersion of a line and it is dense in $T_{r}$.

Case 2: $\alpha / \beta \in \mathbb{Q}$. In this case, the angles of rotation in $T_{r}$ for $\phi_{t}$ are $(2 \alpha+\beta) t$ and $(2 \beta+\alpha) t$. Their quotient is rational; denote it by $p / q$ in reduced form. The orbits are periodic and of slope $p / q$ in $T_{r}$ : they are torus knots of type $(p, q)$, knotted around $C_{1}$ and $C_{2}$. We can see an example in Figure 2. 

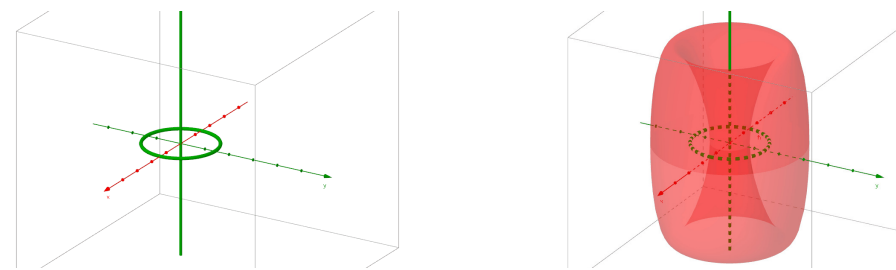

Figure 1. Invariant subsets for an elliptic flow in the Siegel model: invariant $\mathbb{C}$-circles (left), and the invariant torus $T_{4 / 5}$ (right).
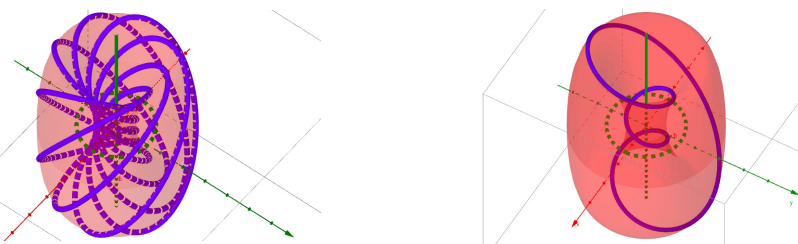

Figure 2. Orbits of elliptic flows in the Siegel model: an orbit for $(2 \alpha+\beta) /(2 \beta+\alpha)=\frac{7}{11}$, a torus knot of type $(7,11)($ left $)$, and an orbit for $(2 \alpha+\beta) /(2 \beta+\alpha)=\frac{1}{3}$, an unknot (right).

Remark 2.15. If $p$ are $q$ are different from \pm 1 , the orbit of a point of $T_{r}$ is a torus knot of type $(p, q)$ and is knotted in $\partial_{\infty} \mathbb{Z}_{\mathbb{C}}^{2}$. If $p$ or $q$ equals \pm 1 , then the orbit is unknotted; we can see an example in Figure 2. This remark will be crucial to identify Dehn surgeries among the structures that we will construct by deformation.

Definition 2.16. Let $n, p$, and $q$ be relatively prime integers with $|p| \geq|q|$. We say that an elliptic element $U \in \mathrm{PU}(2,1)$ is of type $(p / n, q / n)$ if $U$ is conjugate to $E_{\alpha, \beta, \gamma}$ with

$$
\alpha=\frac{2 p-q}{3 n}, \quad \beta=\frac{2 q-p}{3 n}, \quad \text { and } \quad \gamma=-\alpha-\beta=\frac{-p-q}{3 n} .
$$

In this case, $(2 \alpha+\beta) /(2 \beta+\alpha)=p / q$ and the orbits of the flow $\phi_{t}^{\log (U)}$ are its two invariant $\mathbb{C}$-circles and torus knots of type $(p, q)$.

Remark 2.17. (1) Only some elliptic elements are of some type $(p / n, q / n)$. We will see later that elements of some type $(p / n, q / n)$ are the ones for which our construction happens to work.

(2) The trace of an elliptic element gives its three eigenvalues, but it is not enough to determine the type of the element. Indeed, an element of the same trace as an elliptic of type $(p / n, q / n)$ will have the same eigenvalues but not necessarily the same eigenvalue associated to its fixed point in $\mathbb{U}_{\mathbb{C}}^{2}$. Thus, elements of type $(p / n, q / n),(-p / n,(q-p) / n)$, and $((p-q) / n,-q / n)$ have the same trace but are not conjugate. 
Loxodromic flows. Consider a loxodromic element in $\mathrm{SU}(2,1)$ in the Siegel model. Perhaps after a conjugation, we can suppose that it is

$$
T_{\lambda}=\left(\begin{array}{ccc}
\lambda & 0 & 0 \\
0 & \bar{\lambda} / \lambda & 0 \\
0 & 0 & 1 / \bar{\lambda}
\end{array}\right),
$$

where $\lambda \in \mathbb{C}$ is of modulus greater than 1 . We then have $\lambda=r e^{i \alpha}$, with $\alpha \in \mathbb{R}$ and $r>1$. We suppose that $\alpha$ is small enough, so the series $\log \left(T_{\lambda}\right)$ converges. In coordinates $(z, s) \in \mathbb{C} \times \mathbb{R}$, the action of the flow is given by $\phi_{t}^{\log \left(T_{\lambda}\right)}:(z, t) \mapsto\left(\mu_{t} z,\left|\mu_{t}\right|^{2} s\right)$, where $\mu_{t}=r^{t} e^{-3 i \alpha t}$.

Remark 2.18. The flow $\phi_{t}$ fixes globally the points

$$
\left[p_{0}\right]=\left[\begin{array}{l}
0 \\
0 \\
1
\end{array}\right] \text { and }\left[p_{1}\right]=\left[\begin{array}{l}
1 \\
0 \\
0
\end{array}\right]
$$

and stabilizes the $\mathbb{C}$-circle joining them, which is called the axis of $\left[T_{\lambda}\right]$. Furthermore, for all $u \in \partial_{\infty} \mathbb{H}_{\mathbb{C}}^{2}$ not fixed by $T_{\lambda}$,

$$
\lim _{t \rightarrow+\infty} \phi_{t}(u)=\left[p_{1}\right] \quad \text { and } \quad \lim _{t \rightarrow-\infty} \phi_{t}(u)=\left[p_{0}\right] .
$$

In the same way as in the elliptic case, we have flow-invariant objects, related to the centralizer of $T_{\lambda}$.

Remark 2.19. The centralizer of $T_{\lambda}$ is $C\left(T_{\lambda}\right)=\left\{T_{\mu} \mid \mu \in \mathbb{C}^{*}\right\}$. The orbits of this subgroup in $\partial_{\infty} \mathbb{H}_{\mathbb{C}}^{2}$ are the two fixed points of $T_{\lambda}$, the two arcs of the $\mathbb{C}$-circle joining them, and the punctured paraboloids $P_{r}$ for $r \in \mathbb{R}$, as in Figure 3, defined by

$$
P_{r}=\left\{\left[\begin{array}{c}
-\frac{1}{2}\left(|z|^{2}+i s\right) \\
z \\
1
\end{array}\right] \in \partial_{\infty} \mathbb{H}_{\mathbb{C}}^{2} \mid \frac{s}{|z|^{2}}=r\right\} .
$$

Unipotent flows. Consider now a unipotent element of $\mathrm{SU}(2,1)$ in the Siegel model. Perhaps after a conjugation, we can assume that it is, for $(z, s) \in \mathbb{C} \times \mathbb{R}$,

$$
P_{z, s}=\left(\begin{array}{rrc}
1 & -\bar{z} & -\frac{1}{2}\left(|z|^{2}+i s\right) \\
0 & 1 & z \\
0 & 0 & 1
\end{array}\right)
$$

The series $\log \left(P_{z, s}\right)$ converges. In coordinates $(z, s) \in \mathbb{C} \times \mathbb{R}$, the action of the flow is given by $\phi_{t}^{\log \left(P_{z, s}\right)}:\left(z^{\prime}, s^{\prime}\right) \mapsto\left(z^{\prime}+t z, s^{\prime}+t s-2 t \operatorname{Im}\left(\bar{z} z^{\prime}\right)\right)$. In these coordinates, the orbits of the flow are straight lines.

Remark 2.20. If $z=0$, then $\left[P_{z, s}\right]$ is called a vertical parabolic element and all orbits of the flow are vertical lines. If not, then $\left[P_{z, s}\right]$ is called a horizontal parabolic element and the orbits of the flow are lines with different slopes. 

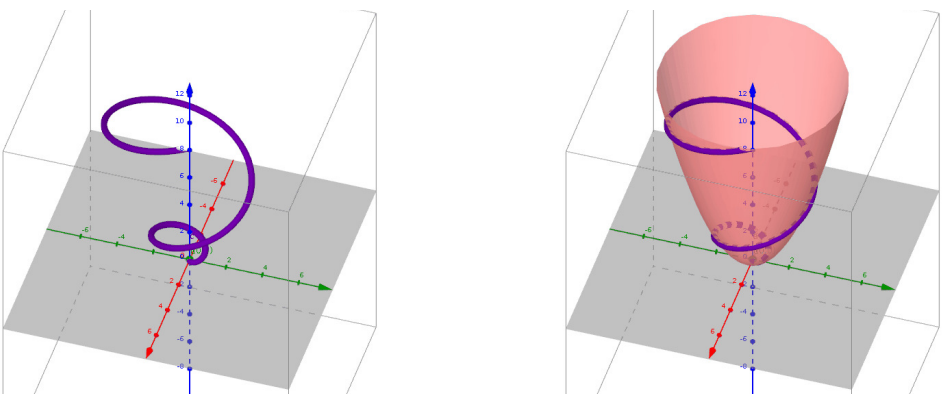

Figure 3. Orbits of loxodromic flows in the Siegel model: an orbit of a loxodromic flow (left) and a cylinder invariant under a loxodromic flow (right).

Some remarks on the convergence of regular elements. The projection

$$
\mathrm{SU}(2,1) \rightarrow \mathrm{PU}(2,1)
$$

is a covering of order 3 ; in order to study the convergence in $\mathrm{PU}(2,1)$ we can focus on the convergence in $\mathrm{SU}(2,1)$.

Let $\left(U_{n}\right)_{n \in \mathbb{N}}$ be a sequence of regular elements of $\mathrm{SU}(2,1)$ converging to $U$ in $\mathrm{SU}(2,1)-\mathbb{C}$ Id. If $U$ is regular, then the convergence is given by the convergence of eigenvectors and eigenvalues. We consider now the case where $U$ is not regular. The continuity of eigenvectors and eigenvalues gives the following lemma.

Lemma 2.21. Suppose that $\left(U_{n}\right)_{n \in \mathbb{N}}$ is a sequence of regular elements of $\mathrm{SU}(2,1)$ converging to $U \in \mathrm{SU}(2,1)-\mathbb{C} \mathrm{Id}$, and let $\left(\left(\left[u_{n}\right], \alpha_{n}\right),\left(\left[v_{n}\right], \beta_{n}\right),\left(\left[w_{n}\right], \gamma_{n}\right)\right)$ be the eigenvectors and eigenvalues of $U_{n}$ in some order. Then, perhaps after relabeling, $\left(\left(\left[u_{n}\right], \alpha_{n}\right),\left(\left[v_{n}\right], \beta_{n}\right),\left(\left[w_{n}\right], \gamma_{n}\right)\right)$ converges to $(([u], \alpha),([v], \beta),([w], \gamma))$ in $\left(\mathbb{C P}^{2} \times \mathbb{C}\right)^{3}$, where $([u], \alpha),([v], \beta),([w], \gamma)$ are (possibly equal) eigenvectors and eigenvalues of $U$.

Consider the case where $U$ is horizontal parabolic. Then, $U$ has a unique fixed point $[p] \in \mathbb{C P}^{2}$, which is in $\partial_{\infty} \mathbb{H}_{\mathbb{C}}^{2}$, and its eigenvalues can be chosen all equal to 1 . Using the above lemma, we deduce that $\left(\alpha_{n}, \beta_{n}, \gamma_{n}\right) \rightarrow(1,1,1)$ and $\left(\left[u_{n}\right],\left[v_{n}\right],\left[w_{n}\right]\right) \rightarrow([p],[p],[p])$. From a geometric point of view on $\mathbb{H}_{\mathbb{C}}^{2} \cup \partial_{\infty} \mathbb{H}_{\mathbb{C}}^{2}$ we make the two following remarks:

Remark 2.22. If the $U_{n}$ are loxodromic of axes $l_{n}$ then the $l_{n}$ leave every compact subset of $\mathbb{H}_{\mathbb{C}}^{2} \cup \partial_{\infty} \mathbb{H}_{\mathbb{C}}^{2}-\{[p]\}$.

Remark 2.23. If the $U_{n}$ are elliptic, they each have two invariant complex geodesics $l_{n}^{(1)}$ and $l_{n}^{(2)}$ (the polar lines $\left[v_{n}\right]^{\perp}$ and $\left[w_{n}\right]^{\perp}$ if $\left[u_{n}\right]$ is the fixed point of $U_{n}$ in $\mathbb{U}_{\mathbb{C}}^{2}$ ). Then the $l_{n}^{(i)}$ leave every compact subset of $\mathbb{M}_{\mathbb{C}}^{2} \cup \partial_{\infty} \mathbb{M}_{\mathbb{C}}^{2}-\{[p]\}$. 
These two remarks will be crucial when understanding the geometry of deformations of spherical CR structures by considering a developing map.

\section{Regular surgeries}

The Ehresmann-Thurston principle. We are going to study spherical CR structures on a 3-manifold $M$. We begin by recalling the formalism of $(G, X)$-structures, that will give us the language to use. In the definitions, $X$ will be a smooth connected manifold and $G$ a subgroup of the diffeomorphisms of $X$ acting transitively and analytically on $X$. We will focus on the case where $X=\partial_{\infty} \mathbb{H}_{\mathbb{C}}^{2}$ and $G=\operatorname{PU}(2,1)$.

Definition 3.1. A $(G, X)$-structure on a manifold $M$ is a pair (Dev, $\rho$ ), up to isotopy, of a local diffeomorphism Dev $: \tilde{M} \rightarrow X$ and a group homomorphism $\rho: \pi_{1}(M) \rightarrow G$ such that for all $x \in \widetilde{M}$ and all $g \in \pi_{1}(M)$ we have $\operatorname{Dev}(g \cdot x)=$ $\rho(g) \cdot \operatorname{Dev}(x)$ for the group actions of $\pi_{1}(M)$ on $\tilde{M}$ and of $G$ on $X$.

We say that Dev is the developing map of the structure and $\rho$ its holonomy.

Remark 3.2. We identify two structures if they are $G$-equivalent, i.e., if there is a $g \in G$ such that the developing maps $\operatorname{Dev}_{1}$ and $\operatorname{Dev}_{2}$ satisfy $\operatorname{Dev}_{2}=g \circ \operatorname{Dev}_{1}$. In this case, the holonomy representations are conjugate and satisfy $\rho_{2}=g \rho_{1} g^{-1}$.

Remark 3.3. The definition we just gave is not the usual one. It is equivalent to the usual definition of a $(G, X)$-structure as an atlas of charts of $M$ taking values in $X$ and whose transition maps are given by elements of $G$. A couple (Dev, $\rho$ ) immediately gives such an atlas, but the construction of (Dev, $\rho$ ) from an atlas requires more work. See, for example, [Thurston 2002]. Nevertheless, we will use both definitions: the first in order to deform a structure, and the second to construct a new one.

We will also sometimes use manifolds with boundary, but the definition of $(G, X)$-structure easily extends to this case. From now on, we consider a compact three-dimensional manifold $M$ with (possibly many) torus boundary components. We are going to study spherical CR structures on $M$, where the model space $X$ is $\partial_{\infty} \mathbb{M}_{\mathbb{C}}^{2}$ and the group $G$ is $\operatorname{PU}(2,1)$.

Definition 3.4. A spherical CR structure is a $\left(\mathrm{PU}(2,1), \partial_{\infty} \mathbb{U}_{\mathbb{C}}^{2}\right)$-structure.

In order to deform the structure using the Ehresmann-Thurston principle that we state below, the essential objects are the representations of $\pi_{1}(M)$ taking values in $\mathrm{PU}(2,1)$.

Notation 3.5. Let $\mathcal{R}\left(\pi_{1}(M), G\right)$ be the set of representations of $\pi_{1}(M)$ taking values in $G$, endowed with the topology of pointwise convergence.

We are going to work with deformations of some structures. In order to state the results on a deformation, we will need to be "far enough from the boundary" or 
"close to the boundary". We are going to consider the union of $M$ with a thickening of its boundary to be able to state the results precisely.

Notation 3.6. If $s \in \mathbb{R}^{+}$, denote by $M_{[0, s[}$ the union of $M$ with a thickening of its boundary. Thus, $M_{[0, s[}=(M \cup(\partial M \times[0, s[)) / \sim$, where we identify $\partial M$ with $\partial M \times\{0\}$. We consider those manifolds as included into each other, in such a way that if $s_{1} \leq s_{2}$, then $M_{\left[0, s_{1}[\right.} \subset M_{\left[0, s_{2}[\right.}$.

Remark 3.7. The manifolds $M_{[0, s[}$ are all homeomorphic to the interior of $M$. We use these cuts in order to get a suitable convergence "far enough" from the boundary of $M$ for geometric structures.

We state the Ehresmann-Thurston principle, which says that we only need to deform in $\mathcal{R}\left(\pi_{1}(M), G\right)$ the holonomy of a $(G, X)$-structure to have a deformation of the structure itself. A proof can be found in [Bergeron and Gelander 2004] or in the survey [Goldman 2010].

Theorem 3.8 (Ehresmann-Thurston principle). Suppose that $M_{[0,1[}$ is endowed with a $(G, X)$-structure of holonomy $\rho_{0}$. For all $\epsilon>0$, if $\rho \in \mathcal{R}\left(\pi_{1}(M), G\right)$ is a deformation close enough to $\rho_{0}$, then there is a $(G, X)$-structure on $M_{[0,1-\epsilon[}$ with holonomy $\rho$ and close to the first structure on $M_{[0,1-\epsilon[}$ in the $\mathcal{C}^{1}$ topology.

Surgeries. As in the real hyperbolic case, we consider Dehn surgeries of $M$, which are, from a topological point of view, a gluing of solid tori on the torus boundaries of $M$. We attempt to extend a spherical CR structure on $M$ to one of its surgeries. We show a result very similar to the one showed by Schwartz [2007], but with some differences. On the one hand, our hypotheses are weaker than Schwartz's and we obtain a geometric structure. On the other hand we do not know if the structure is obtained as a quotient of an open set of $\partial_{\infty} \mathbb{H}_{\mathbb{C}}^{2}$ by the action of a subgroup of $\mathrm{PU}(2,1)$.

Thickenings and lifts. We begin by fixing notation for a torus boundary component, one of its lifts, and the associated peripheral holonomy. We denote by $\tilde{M}$ the universal cover of $M$ and by $\pi: \tilde{M} \rightarrow M$ its covering map. We state all results for a single torus boundary component in order to avoid heavy notation, but the same statements hold for several boundary components.

Notation 3.9. Let $T$ be a fixed torus boundary component of $M$. For $s \in[0,1$ [, let $T_{s}=T \times\{s\} \subset M_{[0,1[}$, and, for an interval $I \subset[0,1[$, let

$$
T_{I}=\bigcup_{s \in I} T_{s}=T \times I \subset M_{[0,1[} .
$$

Let $\widetilde{T}_{[0,1[}$ denote some connected component of $\pi^{-1}\left(T_{[0,1[}\right) \subset \widetilde{M}_{[0,1[}$ : it is a universal cover of $T_{[0,1[}$ embedded in $\widetilde{M}_{[0,1[}$. Finally, for $s \in\left[0,1\left[\right.\right.$, set $\widetilde{T}_{s}=\pi^{-1}\left(T_{s}\right) \cap \widetilde{T}_{[0,1[}$ and, for an interval $I \subset\left[0,1\left[\right.\right.$, set $\widetilde{T}_{I}=\bigcup_{s \in I} \widetilde{T}_{s}$.

We make some remarks on the choices made by using this notation: 
Remark 3.10. For all $s \in\left[0,1\left[\right.\right.$, we see that $\widetilde{T}_{s}$ is homeomorphic to $\mathbb{R}^{2}$. Furthermore, $\widetilde{T}_{I}$ is homeomorphic to $\mathbb{R}^{2} \times I$.

Remark 3.11. The choice of $\widetilde{T}_{[0,1[}$ fixes an injection of the fundamental group of $T$ into the fundamental group of $M$ by identifying $\pi_{1}(T)$ with the stabilizer of $\widetilde{T}_{[0,1[}$ for the action of $\pi_{1}(M)$ on $\tilde{M}_{[0,1[}$. In the rest of the paper, we will use additive notation for $\pi_{1}(T) \simeq \mathbb{Z}^{2}$, in order to use the standard notations and tools for a group isomorphic to $\mathbb{Z}^{2}$. Nevertheless, the identification of $\pi_{1}(T)$ with a subgroup of $\pi_{1}(M)$ will lead to a slight abuse of notation: we will keep multiplicative notation for $\pi_{1}(M)$, but when considering elements of $\pi_{1}(T)$ we will use additive notation.

Notation 3.12. With the fixed injection of $\pi_{1}(T)$ into $\pi_{1}(M)$, by restricting the holonomy $\rho$ of a $(G, X)$-structure we have a peripheral holonomy $h_{\rho}: \pi_{1}(T) \rightarrow G$.

Notation 3.13. We denote by $\mathcal{R}_{1}\left(\pi_{1}(M), G\right) \subset \mathcal{R}\left(\pi_{1}(M), G\right)$ the set of representations $\rho$ such that the image of $h_{\rho}$ is generated by a single element. When $\rho \in \mathcal{R}_{1}\left(\pi_{1}(M), \mathrm{PU}(2,1)\right)$ has $[U] \in \mathrm{PU}(2,1)$ as a preferred generator for its image, we write $\phi_{t}^{\rho}$ for $\phi_{t}^{\log ([U])}$.

Horotubes. We use the definitions related to horotubes given in [Schwartz 2007]:

Definition 3.14. Let $[P] \in \mathrm{PU}(2,1)$ be a parabolic element with fixed point $p \in \partial_{\infty} \mathbb{H}_{\mathbb{C}}^{2}$. A $[P]$-horotube is an open set $H$ of $\partial_{\infty} \mathbb{H}_{\mathbb{C}}^{2}-\{p\}$, invariant under $[P]$ and such that the complement of $H /\langle[P]\rangle$ in $\left(\partial_{\infty} \mathbb{H}_{\mathbb{C}}^{2}-\{p\}\right) /\langle[P]\rangle$ is compact.

In order to work with more regular objects, we often ask horotubes to be nice:

Definition 3.15. A $[P]$-horotube $H$ is nice if $\partial H$ is a smooth cylinder invariant by the flow $\phi_{t}^{\log ([P])}$.
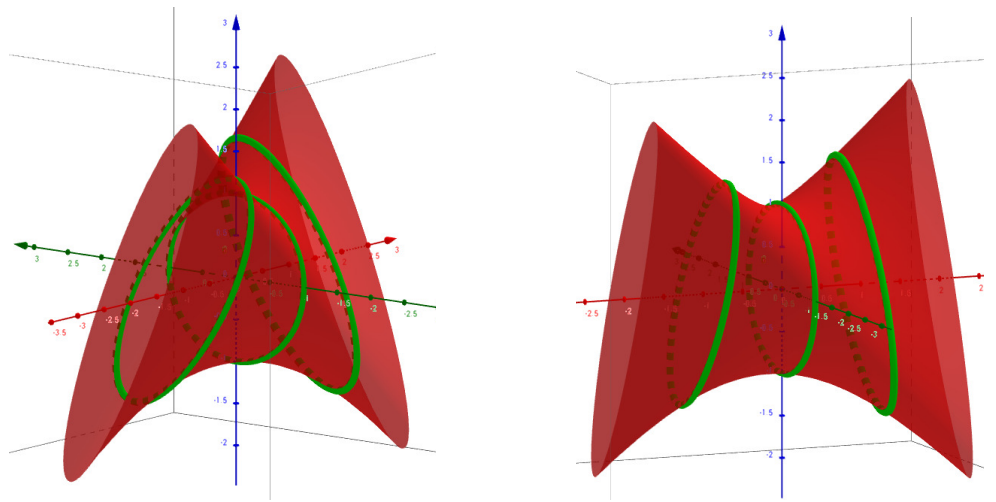

Figure 4. The boundary of a nice horotube in the Siegel model. The horotube is outside the red surface. 
Remark 3.16. If $H$ is a nice [P]-horotube, then $\partial H$ is the orbit for $\phi_{t}^{\log ([P])}$ of an embedded circle of $\partial_{\infty} \mathbb{H}_{\mathbb{C}}^{2}-\{p\}$. We can see an example in Figure 4.

Shrinking the horotube if necessary, we may assume it is nice:

Lemma 3.17 [Schwartz 2007, Chapter 7]. Let $H$ be a [P]-horotube. Then, there is a nice $[P]$-horotube $H^{\prime}$ such that $H^{\prime} \subset H$ and $\left(H-H^{\prime}\right) /\langle[P]\rangle$ is of compact closure in $\left(\partial_{\infty} \mathbb{H}_{\mathbb{C}}^{2}-\{p\}\right) /\langle[P]\rangle$.

From now on, we suppose that $M_{[0,1[}$ has a spherical CR structure with developing map $\operatorname{Dev}_{0}$ and holonomy $\rho_{0}$. We also make two more hypotheses:

(1) The image of the peripheral holonomy $h_{\rho_{0}}$ is unipotent of rank 1 and generated by an element $\left[U_{0}\right] \in \mathrm{PU}(2,1)$.

(2) There is $s \in\left[0,1\left[\right.\right.$ such that $\operatorname{Dev}_{0}\left(\widetilde{T}_{[s, 1[}\right)$ is a $\left[U_{0}\right]$-horotube.

Marking of $\pi_{1}(T)$. We are going to fix a marking of $\pi_{1}(T)$ naturally deduced from the structure given by $\operatorname{Dev}_{0}$ and $\rho_{0}$. This marking will be useful to identify the Dehn surgeries obtained when deforming the structure. It is essentially the same marking as the one given in [Schwartz 2007, Chapter 8]; its definition uses the two hypotheses given above.

Notation 3.18. Fix $s^{\prime} \in\left[s, 1\left[\right.\right.$ and $x_{0} \in \operatorname{Dev}_{0}\left(T_{s^{\prime}}\right)$. Let $l$ be the loop given by the projection of $t \mapsto \phi_{t}^{\rho_{0}}\left(x_{0}\right)$. As $h_{\rho_{0}}(l)=\left[U_{0}\right]$ generates the image of $h_{\rho_{0}}$ and since a unipotent subgroup of $\mathrm{PU}(2,1)$ has no torsion, $l$ is a primitive element of $\pi_{1}(T)$.

Notation 3.19. Since $h_{\rho_{0}}$ is unipotent of rank 1 and a unipotent subgroup of $\mathrm{PU}(2,1)$ has no torsion, its kernel is generated by a primitive element $m$. We orient $m$ in such a way that $(l, m)$ is a direct basis of $\pi_{1}(T)$ (for the orientation given by the inside normal in the horotube).

Remark 3.20. The definition of $l$ and $m$ does not depend on the choice of $s^{\prime}$ nor of $x_{0}$. Nevertheless, we make a choice for orientations. The one for $m$ is explicit, but the orientation of $l$ is given by the choice of $\left[U_{0}\right]$ or $\left[U_{0}\right]^{-1}$ as a generator for the image of $h_{\rho_{0}}$.

Remark 3.21. Schwartz [2007] gives a "canonical" choice for the orientations of $l$ and $m$ (denoted $\beta$ and $\alpha$ ). It is almost the same choice as the one made above, but he has a preferred choice for $\left[U_{0}\right]$. Note that the marking $(l, m)$ given here might not be the usual one. If we have another marking of $\pi_{1}(T)$, for example when $M$ is a knot complement, changing markings can be done easily when $\rho_{0}$ is known explicitly.

Definition 3.22. For two relatively prime integers $p, q$, we denote by $M^{(p, q)}$ the manifold obtained by gluing a solid torus $D^{2} \times S^{1}$ on the boundary $T$ of $M$ such that the loop $p l+q m$ of $T$ becomes trivial in $D^{2} \times S^{1}$. We refer to it as the Dehn surgery of $M$ of type $(p, q)$ or of slope $p / q$. 


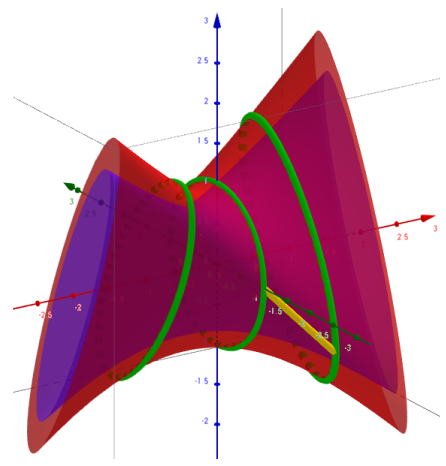

Figure 5. The curve $m$ (in green) and the curve $l$ (in yellow) in the image of $\operatorname{Dev}_{0}\left(\widetilde{T}_{S^{\prime}}\right)$.

In the real hyperbolic case, deforming the complete hyperbolic structure on $M$ gives structures on all but a finite number of Dehn surgeries $M^{(p, q)}$ of $M$, as is shown in [Thurston 2002]. The main idea to prove it is to deform the structure "far" from the cusp, cut by $T$, look at the developing map near the boundary $T$, and then notice that a solid torus can be glued to this boundary. What follows, stated in the spherical CR case, is inspired by this technique. The deformation "far" from the cusp gives rise to a developing map near $T$, and the manifolds that can be glued are solid tori only in some cases.

A surgery theorem. We are now able to state a spherical CR surgery theorem. It says that in a neighborhood of the structure $\left(\operatorname{Dev}_{0}, \rho_{0}\right)$, under some discreteness conditions, spherical CR structures on $M$ come from structures on Dehn surgeries of $M$, and in some cases another kind of surgery.

Theorem 3.23. Let $M$ be a three-dimensional compact manifold with torus boundary components. Let $T$ be one boundary torus of $M$. Suppose that there is a spherical CR structure $\left(\operatorname{Dev}_{0}, \rho_{0}\right)$ on $M_{[0,1[}$ such that:

(1) The image of the peripheral holonomy $h_{\rho_{0}}$ corresponding to $T$ is unipotent of rank 1 and generated by an element $\left[U_{0}\right] \in \mathrm{PU}(2,1)$.

(2) There is $s \in\left[0,1\left[\right.\right.$ such that $\operatorname{Dev}_{0}\left(\widetilde{T}_{[s, 1[}\right)$ is a $\left[U_{0}\right]$-horotube.

Then there is an open set $\Omega$ of $\mathcal{R}_{1}\left(\pi_{1}(M), \mathrm{PU}(2,1)\right)$ containing $\rho_{0}$ such that, for all $\rho \in \Omega$ for which the image of $h_{\rho}$ is generated by a single element $[U] \in \mathrm{PU}(2,1)$, there is a spherical CR structure on $M$ with holonomy $\rho$. Furthermore, for the marking $(l, m)$ of $\pi_{1}(T)$ described above:

(1) If $[U]$ is loxodromic, then the structure extends to a spherical CR structure on the Dehn surgery of $M$ of type $(0,1)$. 
(2) If [U] is elliptic of type $(p / n, \pm 1 / n)$, then the structure extends to a spherical CR structure on the Dehn surgery of $M$ of type $(n, \pm p)$.

(3) If [U] is elliptic of type $(p / n, q / n)$ with $|p|,|q|>1$, then the structure extends to a spherical CR structure on the gluing of $M$ with a compact manifold with torus boundary $V(p, q, n)$. Furthermore $V(p, q, n)$ is a torus knot complement in the lens space $L(n, \alpha)$, where $\alpha \equiv p^{-1} q \bmod n$.

Remark 3.24. The existence of the spherical CR structure on $M$ is a consequence of the Ehresmann-Thurston principle. To extend the structure we need a local surgery result, similar to the one given in [Schwartz 2007], and which is given in Section 5.

Remark 3.25. If [ $U$ ] is parabolic, the theorem still holds, but the spherical CR structure extends to a thickening of $M$ that is homeomorphic to $M$ itself. We also exclude from the discussion the case where $[U]$ is elliptic with irrational angle, for which there is no reasonable filling for the structure, and the case where $[U]$ is nonregular elliptic, for which the techniques used to prove Theorem 3.23 do not apply.

\section{Deformations of the Deraux-Falbel structure on the figure-eight knot complement}

We are going to apply Theorem 3.23 in the case of the spherical CR structure on the figure-eight knot given in [Deraux and Falbel 2015]. We will use some results of [Deraux 2014], where Deraux describes a Ford domain for the structure, and also some results of [Falbel et al. 2016], where the authors describe the irreducible components of the $\mathrm{SL}_{3}(\mathbb{C})$ character variety of the figure eight knot complement.

Notation 4.1. In the rest of this section, we denote by $M$ the figure-eight knot complement.

The Deraux-Falbel structure. We begin by recalling quickly the results in [Deraux and Falbel 2015]. In that paper, the fundamental group of $M$ is given by

$$
\pi_{1}(M)=\left\langle g_{1}, g_{2}, g_{3} \mid g_{2}=\left[g_{3}, g_{1}^{-1}\right], g_{1} g_{2}=g_{2} g_{3}\right\rangle .
$$

The authors construct a uniformizable spherical CR structure on $M$ with unipotent peripheral holonomy. The holonomy representation $\rho_{0}$ is given by

$\rho_{0}\left(g_{1}\right)=\left[G_{1}\right]=\left[\begin{array}{ccc}1 & 1 & -\frac{1}{2}-\frac{\sqrt{7}}{2} i \\ 0 & 1 & -1 \\ 0 & 0 & 1\end{array}\right] \quad$ and $\quad \rho_{0}\left(g_{3}\right)=\left[G_{3}\right]=\left[\begin{array}{ccc}1 & 0 & 0 \\ -1 & 1 & 0 \\ -\frac{1}{2}+\frac{\sqrt{7}}{2} i & 1 & 1\end{array}\right]$.

Remark 4.2. This representation is in the component $R_{2}$ of the character variety of [Falbel et al. 2016]. For the notation from Section 5.2 of that reference, we have $A=g_{3}$ and $B=g_{1}$. With this notation, the usual longitude-meridian pair $\left(l_{0}, m_{0}\right)$ 
of the knot complement satisfies

$$
m_{0}=g_{3} \quad \text { and } \quad l_{0}=g_{1}^{-1} g_{3} g_{1} g_{3}^{-2} g_{1} g_{3} g_{1}^{-1} .
$$

Furthermore, we check easily that $\rho_{0}\left(m_{0}\right)^{3}=\rho_{0}\left(l_{0}\right)$, so $\rho_{0}\left(3 m_{0}-l_{0}\right)=\mathrm{Id}$.

Notation 4.3. From now on, in order to have the same notation as [Deraux 2014], we consider the pair $\left(l_{1}, m_{1}\right)$ obtained by conjugation by $g_{2}$, so that $m_{1}=g_{2} g_{3} g_{2}^{-1}=g_{1}$.

Let $l=m_{0}$ and $m=3 m_{0}-l_{0}$. In this way, $m$ generates $\operatorname{ker}\left(\rho_{0}\right)$ and $\rho(l)$ generates $\operatorname{Im}\left(\rho_{0}\right)$ : this is a marking as in the one on page 269.

Checking the hypotheses. Recall the hypotheses of Theorem 3.23:

(1) The peripheral holonomy $h_{\rho_{0}}$ is unipotent with image generated by a single element $\left[U_{0}\right] \in \mathrm{PU}(2,1)$.

(2) There exists $s \in\left[0,1\left[\right.\right.$ such that $\operatorname{Dev}_{0}\left(\widetilde{T}_{[s, 1[}\right)$ is a $\left[U_{0}\right]$-horotube.

The first hypothesis is satisfied by the Deraux-Falbel structure: the peripheral holonomy is unipotent, its image is generated by $\left[G_{1}\right]=\rho_{0}(l)$ and $\rho_{0}(m)=[\mathrm{Id}]$.

In order to check the second hypothesis, we use the results of [Deraux 2014]. In that paper, Deraux finds with a different technique the Deraux-Falbel structure [2015]. He considers a Ford domain $F$ in $\mathbb{H}_{\mathbb{C}}^{2}$ for $\Gamma=\rho_{0}\left(\pi_{1}(M)\right.$ ) (Theorem 5.1) and then studies its boundary at infinity in $\partial_{\infty} \mathbb{H}_{\mathbb{C}}^{2}$ (Section 8). The manifold $M$ is then obtained as a quotient of a $G_{1}$-invariant domain $E=\partial_{\infty} F$, that is, in $\partial_{\infty} \mathbb{H}_{\mathbb{C}}^{2} \simeq(\mathbb{C} \times \mathbb{R}) \cup\{\infty\}$, the exterior of a $G_{1}$-invariant cylinder $C$ embedded in $\mathbb{C} \times \mathbb{R}$ (Proposition 8.1). The domain $E$ is a $\left[G_{1}\right]$-horotube; so there exists $s \in[0,1$ [ such that the image by the developing map of $\widetilde{T}_{[s, 1[}$ is a $\left[G_{1}\right]$-horotube contained in $E$. Thus, the second hypothesis is satisfied.

So, the conclusion of Theorem 3.23 holds. By changing coordinates in order to have the usual marking for the fundamental group of the boundary of $M$, we get:

Proposition 4.4. There is an open set $\Omega$ of $\mathcal{R}_{1}\left(\pi_{1}(M), \mathrm{PU}(2,1)\right)$ such that, for all $\rho \in \Omega$ such that the image of $h_{\rho}$ is generated by an element $[U] \in \mathrm{PU}(2,1)$, there exists a spherical CR structure on $M$ of holonomy $\rho$. Furthermore, for the usual marking $\left(l_{0}, m_{0}\right)$ of $\pi_{1}(T)$ :

(1) If $[U]$ is loxodromic, then the structure extends to a spherical CR structure on the Dehn surgery of type $(-1,3)$ of $M$.

(2) If $[U]$ is elliptic of type $(p / n, \pm 1 / n)$, then the structure extends to a spherical CR structure on the Dehn surgery of type $(-n, \pm p+3 n)$ of $M$.

(3) If $[U]$ is elliptic of type $(p / n, q / n)$ with $|p|,|q|>1$, then the structure extends to a spherical CR structure on the gluing of $M$ to a compact manifold with torus boundary $V(p, q, n)$ along their boundaries. Furthermore, $V(p, q, n)$ is the complement of a torus knot in the lens space $L(n, \alpha)$, where $\alpha \equiv p^{-1} q \bmod n$. 
Remark 4.5. If $[U]$ is parabolic, then the theorem still holds, but the spherical CR structure extends to a thickening of $M$. These structures are given in [Deraux 2014].

Remark 4.6. We wonder if Schwartz's horotube surgery theorem [2007, Theorem 1.2] can be applied in this case. For $\Gamma=\rho_{0}\left(\pi_{1}(M)\right)$, the construction of Deraux and Falbel [2015] states that the regular set $\Omega_{\Gamma}$ is nonempty and that $\Omega_{\Gamma} / \Gamma$ is homeomorphic to $M$, but we do not have any more information about $\Omega_{\Gamma}$ and the limit set $\Lambda_{\Gamma}=\partial_{\infty} \mathbb{Z}_{\mathbb{C}}^{2}-\Omega_{\Gamma}$. In order to apply the horotube surgery theorem, we would have to check several nontrivial hypotheses. In particular we do not know how to prove that the set $\Lambda_{\Gamma}$ is porous. One of the main motivations of this paper was to state a result with more simple hypotheses, even if we obtain weaker conclusions when both theorems can be applied.

Deformations of the structure. It remains to see that the open set $\Omega \subset \mathcal{R}_{1}\left(\pi_{1}(M)\right)$ is not reduced to a point to get interesting conclusions. The representation $\rho_{0}$ is in the component $R_{2}$ of the $\mathrm{SL}_{3}(\mathbb{C})$-character variety described in [Falbel et al. 2016]. In Section 5 of that paper, the representations in $R_{2}$ taking values in $\mathrm{SU}(2,1)$ are parametrized up to conjugacy, at least in a neighborhood of $\rho_{0}$, by a complex parameter $u=\operatorname{tr}\left(\rho\left(m_{0}\right)\right)$. We denote by $G(u)=\rho\left(m_{0}\right)$ the corresponding matrix.

Setting $v=\bar{u}, \Delta=4 u^{3}+4 v^{3}-u^{2} v^{2}-16 u v+16$, and

$$
\Delta^{\prime}=\frac{-16+8 u v-2 v^{3}-4 \sqrt{\Delta}}{8 u^{2}-6 u v^{2}+v^{4}},
$$

the parametrization is explicitly given by

$$
\left[G_{3}^{-1}(u)\right]=\rho(a)=\left[\begin{array}{ccc}
\frac{1}{2} v & 1 & -(1-i) \Delta^{\prime} \\
\frac{1}{8}(1+i)\left(-2 u+v^{2}\right) & \frac{1}{4}(1+i) v & 1 \\
\frac{1}{16}\left(8-4 u v+v^{3}-2 \sqrt{\Delta}\right) & \frac{1}{8}\left(-4 u+v^{2}\right) & \frac{1}{4}(1-i) v
\end{array}\right]
$$

and

$$
\left[G_{1}^{-1}(u)\right]=\rho(b)=\left[\begin{array}{ccc}
\frac{1}{2} v & i & (1+i) \Delta^{\prime} \\
-\frac{1}{8}(1+i)\left(-2 u+v^{2}\right) & \frac{1}{4}(1-i) v & i \\
-\frac{1}{16}\left(8-4 u v+v^{3}-2 \sqrt{\Delta}\right) & -\frac{i}{8}\left(-4 u+v^{2}\right) & \frac{1}{4}(1+i) v
\end{array}\right] .
$$

Recall that for this choice of generators the usual meridian $m_{0}$ is given by $m_{0}=a^{-1}$. The Hermitian form preserved by this representation is given by the matrix ${ }^{2}$

$$
H=\left(\begin{array}{ccc}
\frac{1}{8}(\Delta-16)\left(\sqrt{\Delta}+|u|^{2}-4\right) & 0 & 0 \\
0 & 16-\Delta & 0 \\
0 & 0 & 8(\sqrt{\Delta}+4)
\end{array}\right) \text {. }
$$

${ }^{2}$ We write here the opposite of the matrix $H$ appearing in [Falbel et al. 2016] in order to have signature $(2,1)$ and not $(1,2)$. 


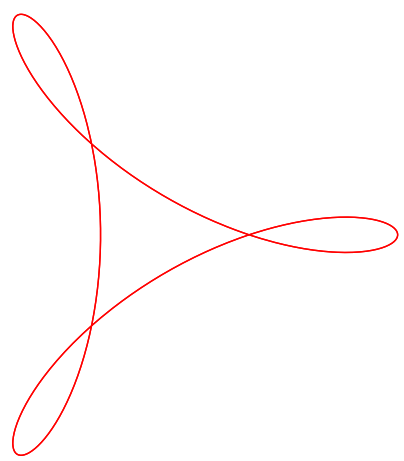

Figure 6. Domain parametrizing a component of the deformation variety near $\rho_{0}$.

Furthermore, in the whole component the relation $\rho\left(l_{0}\right)=\rho\left(m_{0}\right)^{3}$ holds, so $\mathcal{R}_{1}\left(\pi_{1}(M)\right) \cap R_{2}=\mathcal{R}\left(\pi_{1}(M)\right) \cap R_{2}$. By projecting to $\mathrm{PU}(2,1)$, we can apply Theorem 3.23 on an open set containing $3=\operatorname{tr}\left(\rho_{0}\left(m_{0}\right)\right)$ with these parameters.

Figure 6, taken from [Falbel et al. 2016], shows an open set of $\mathbb{C}$ where we have representations. By noting $\operatorname{tr}\left(\rho_{0}\left(m_{0}\right)\right)=x+i y$, the component containing $\rho_{0}$ admits as parameters the regions with boundary the curve $\Delta(x, y)=0$ and containing the points $3,3 \omega$, and $3 \omega^{2}$, where

$$
\Delta(x, y)=-x^{4}-y^{4}-2 x^{2} y^{2}-24 x y^{2}+8 x^{3}-16 x^{2}-16 y^{2}+16 .
$$

Now let us plot the curve $\mathcal{C}$ of traces of nonregular elements of $\mathrm{SU}(2,1)$. It is given by the zeroes of the function $f(z)=|z|^{4}-8 \operatorname{Re}\left(z^{3}\right)+18|z|^{2}-27$ (see Proposition 2.9). The curve separates regular elliptic and loxodromic elements. It has a singularity at the point $u=3$ : thus a neighborhood of this point contains points corresponding to representations where the peripheral holonomy is loxodromic and points where it is regular elliptic.

Remark 4.7. The parabolic deformations of the Deraux-Falbel structure given in [Deraux 2014] correspond to the points of $\mathcal{C}$.

We can therefore apply the first point of Proposition 4.4 to the space of holonomy representations given by the parameters above. We obtain the following proposition:

Proposition 4.8. There exist infinitely many spherical CR structures on the Dehn surgery of $M$ of type $(-1,3)$.

Remark 4.9. This surgery is the unit tangent bundle to the hyperbolic orbifold $(3,3,4)$. It is a Seifert manifold of type $S^{2}(3,3,4)$. See, for example, Chapter 5 of the book of Cooper, Hodgson, and Kerckhoff [Cooper et al. 2000] or the paper [Deraux 2015]. Deraux [2014, Section 4; 2015, Theorem 4.2] also remarks that the image of $\rho_{0}$ is a faithful representation of the even words of the $(3,3,4)$ triangle 
group, generated by involutions $I_{1}, I_{2}, I_{3}$. This identification satisfies $G_{1}=I_{2} I_{3} I_{2} I_{1}$, $G_{2}=I_{1} I_{2}, G_{3}=I_{2} I_{1} I_{2} I_{3}$, and the triangle group relations: $\left(G_{2}\right)^{4}=\left(I_{1} I_{2}\right)^{4}=\mathrm{Id}$, $\left(G_{1} G_{2}\right)^{3}=\left(I_{2} I_{3}\right)^{3}=\mathrm{Id}$, and $\left(G_{2} G_{1} G_{2}\right)^{3}=\left(I_{1} I_{3}\right)^{3}=\mathrm{Id}$. Furtheremore, the image of the usual meridian $m_{0}$ is $G_{3}$.

This group is the fundamental group of a Seifert manifold of type $S^{2}(3,3,4)$. Since the relation $l_{0}=m_{0}^{3}$ holds in the whole component $R_{2}$, the images of representations in $R_{2}$ are representations of this index-2 subgroup of the $(3,3,4)$ triangle group. Furthermore, Parker, Wang, and Xie [Parker et al. 2016] show that a $\mathrm{PU}(2,1)$ representation of the $(3,3,4)$ triangle group is discrete and faithful if and only if the image of $I_{1} I_{3} I_{2} I_{3}$ is nonelliptic. Note that $G_{1} I_{1} I_{3} I_{2} I_{3}=\left(I_{2} I_{3}\right)^{3}=\mathrm{Id}$, so the representation of the triangle group is discrete and faithful if and only if the corresponding peripheral holonomy is nonelliptic. They also give a one-parameter family of such representations, corresponding to the parameters $u \in \mathbb{R}$. Thus, there exists $\delta>0$ such that all the spherical CR structures on the Dehn surgery of $M$ of type $(-1,3)$ with parameter $u$ in the interval $] 3,3+\delta[$ have discrete and faithful holonomy.

Since the parameter is the trace of an element, we know that cases (2) and (3) of Proposition 4.4 happen infinitely many times, but we can not distinguish at first sight, for a given trace, if it is a Dehn surgery or a gluing of a $V(p, q, n)$ manifold. Nevertheless, using a computation with the explicit parametrization of [Falbel et al. 2016] and the continuity of eigenvalues we prove:

Proposition 4.10. There is $\delta>0$ such that, if $p, n \in \mathbb{N}$ are relatively prime integers such that $p / n<\delta$, then the Dehn surgery of $M$ of type $(-n,-p+3 n)$ admits a spherical CR structure.

Proof. Let $p, n \in \mathbb{N}$ be relatively prime integers. Let

$$
\alpha=\frac{-2 p-1}{3 n}, \quad \beta=\frac{2+p}{3 n}, \quad \gamma=\frac{p-1}{3 n}, \quad \text { and } \quad u=e^{i \alpha}+e^{i \beta}+e^{i \gamma} .
$$
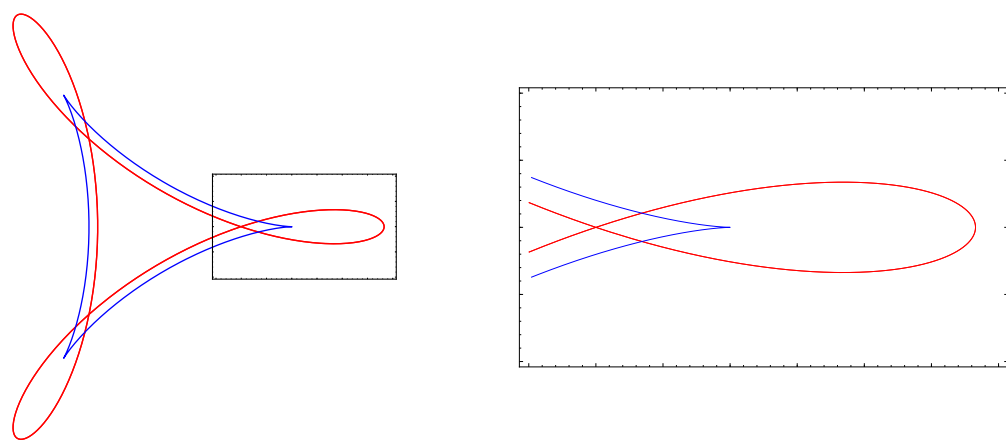

Figure 7. Curve of nonregular elements in a component of the deformation variety near $\rho_{0}$ (left) and detail of same (right). 
We only need to show that if $p / n$ is small enough, the eigenvalue of $\rho(m)=G_{3}^{-1}(u)$ corresponding to a negative eigenvector is $e^{i \gamma}$, and so $G_{3}(u)$ is of type $(p / n,-1 / n)$.

Since eigenvectors and eigenvalues are continuous functions of $u$ in the connected component of regular elliptics, in $R_{2}$, as in Figure 7, the statement is true for all $(p, n)$ if and only if it is true for a particular choice of $(p, n)$. For the arbitrary choice $(p, n)=(3,23)$ an explicit computation shows that $G_{3}(u)$ is of type $\left(\frac{3}{23},-\frac{1}{23}\right)$.

\section{Proof of Theorem 3.23}

In this section, we are going to prove Theorem 3.23. We use the notation of Section 3. We have a manifold $M$ with a torus boundary $T$, endowed with a spherical CR structure $\left(\operatorname{Dev}_{0}, \rho_{0}\right)$ such that the image of the holonomy $h_{\rho_{0}}$ is unipotent of rank 1 and generated by an element $\left[U_{0}\right] \in \mathrm{PU}(2,1)$. We suppose that there is $s \in[0,1[$ such that $\operatorname{Dev}_{0}\left(\widetilde{T}_{[s, 1[}\right)$ is a $\left[U_{0}\right]$-horotube. Recall that we work with a single boundary component $T$ to avoid heavy notation, but the proof works for several boundary components.

In order to prove the theorem, we begin by rewriting the hypotheses to make them easier to handle. The existence of a spherical CR structure on $M$ for a deformation of $\rho_{0}$ will be a consequence of the Ehresmann-Thurston principle. To extend it to a surgery of $M$, we need only a local surgery result by looking near the boundary of $M_{[0,1[}$. This surgery result is very similar, in cases (1) and (2), to the one given in [Schwartz 2007, Chapter 8].

Rewriting the hypotheses. First of all, we rewrite the second hypothesis. Fix a diffeomorphism $\psi: \mathbb{R}^{2} \times\left[0,1\left[\rightarrow \widetilde{T}_{[0,1[}\right.\right.$, such that:

(1) $\psi\left(\mathbb{R}^{2} \times\{s\}\right)=\widetilde{T}_{s}$ for all $s \in[0,1[$.

(2) $\psi$ induces a diffeomorphism between $\mathbb{R} \times S^{1} \times\left[0,1\left[\right.\right.$ and $\widetilde{T}_{[0,1[} / \operatorname{ker}\left(h_{\rho_{0}}\right)$.

To avoid too much notation, we identify $\mathbb{R}^{2} \times\left[0,1\left[\right.\right.$ with $\widetilde{T}_{[0,1[}$ and $\mathbb{R} \times S^{1} \times[0,1[$ with $\widetilde{T}_{[0,1[} / \operatorname{ker}\left(h_{\rho_{0}}\right)$. In this case, the developing map Dev $\operatorname{Din}_{0}$ induces a diffeomorphism between $\widetilde{T}_{[0,1[} / \operatorname{ker}\left(h_{\rho_{0}}\right)$ and $\operatorname{Dev}_{0}\left(\widetilde{T}_{[0,1[}\right)$ that we will still call $\operatorname{Dev}_{0}$. We replace hypothesis (2) of the theorem by hypotheses (2') and (3) described below:

Hypothesis $\left(2^{\prime}\right)$ : There are $0<s_{1}<s_{2}<1$ such that:

(1) $\operatorname{Dev}_{0}\left(\{0\} \times S^{1} \times\{s\}\right)$ is a circle transverse to the flow for all $s \in\left[s_{1}, s_{2}\right]$.

(2) $\operatorname{Dev}_{0}(t, \zeta, s)=\phi_{t}^{\rho_{0}}\left(\operatorname{Dev}_{0}(0, \zeta, s)\right)$ for all $(t, \zeta, s) \in \mathbb{R} \times S^{1} \times\left[s_{1}, s_{2}\right]$.

Remark 5.1. Thanks to Lemma 3.17, it is clear that hypothesis $\left(2^{\prime}\right)$ follows from hypothesis (2). Perhaps after considering an isotopy and slightly increasing $s$, we can suppose that the horotube $\operatorname{Dev}_{0}\left(\widetilde{T}_{[s, 1[}\right)$ is nice. We only need then to consider the restriction to a segment $\widetilde{T}_{\left[s_{1}, s_{2}\right]}$. 

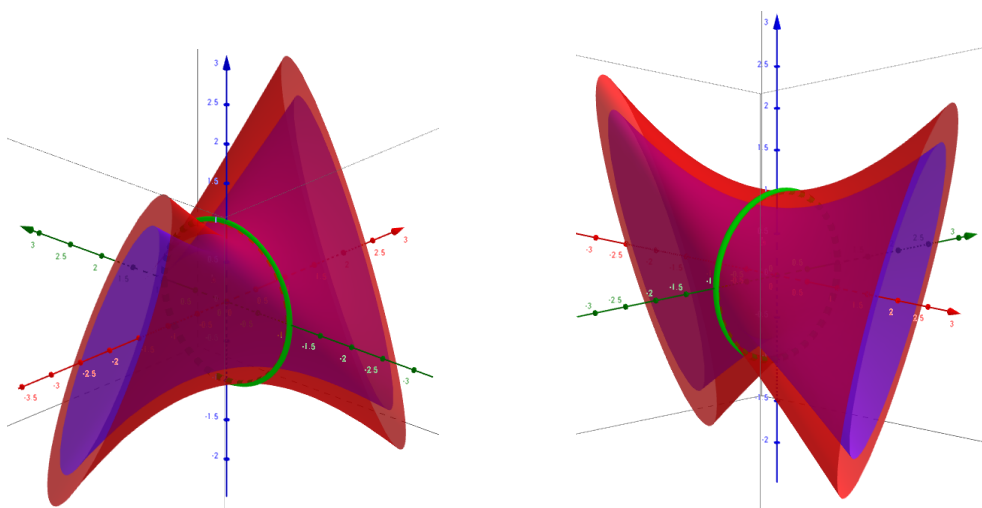

Figure 8. Two views of surfaces bounding a region of the form $\operatorname{Dev}_{0}\left(\widetilde{T}_{\left[s_{1}, s_{2}\right]}\right)$.

Hypothesis $\left(2^{\prime}\right)$ gives, in particular, that $\operatorname{Dev}_{0}\left(\widetilde{T}_{s_{2}}\right)$ separates $\partial_{\infty} \mathbb{H}_{\mathbb{C}}^{2}-\{p\}$ in two connected components: a solid cylinder $C_{S_{2}}$ and the exterior of this cylinder, which is homeomorphic to $\left.S^{1} \times \mathbb{R} \times\right] 0,+\infty[$. Hypothesis (3) tells us that the structure of $M$ is on the correct side of the tube:

Hypothesis (3): $\operatorname{Dev}_{0}\left(\widetilde{T}_{s_{1}}\right)$ is contained in $C_{S_{2}}$.

Remark 5.2. Hypothesis (2) is equivalent to hypotheses $\left(2^{\prime}\right)$ and (3). The implication from (2) to $\left(2^{\prime}\right)$ and (3) is clear, and, if we suppose $\left(2^{\prime}\right)$ and (3), the structure can be extended to the outside in such a way that $\operatorname{Dev}_{0}\left(\widetilde{T}_{\left[s_{2}, 1[\right.}\right)$ is the horotube with boundary $\operatorname{Dev}_{0}\left(\widetilde{T}_{S_{2}}\right)$.

Deforming the structure. We now prove Theorem 3.23. To begin, assume that the rewritten hypotheses on page 276 are satisfied. Let $\rho$ be a deformation close to $\rho_{0}$ in $\mathcal{R}_{1}\left(\pi_{1}(M), \mathrm{PU}(2,1)\right)$ such that $h_{\rho}(m)=\mathrm{Id}$. The image of $h_{\rho}$ is then generated by $[U]=\rho(l)$. We suppose that $[U]$ is a regular element.

Let $\epsilon>0$. By the Ehresmann-Thurston principle, if $\rho$ is close enough to $\rho_{0}$, there is a spherical CR structure on $M_{\left[0, s_{2}+\epsilon[\right.}$ with holonomy map $\rho$. We then have a developing map $\operatorname{Dev}_{\rho}: \widetilde{M}_{\left[0, s_{2}+\epsilon\right]} \rightarrow \partial_{\infty} \mathbb{U}_{\mathbb{C}}^{2}$ close to $\operatorname{Dev}_{0}$ in the $\mathcal{C}^{1}$ topology. So, we can suppose that $\operatorname{Dev}_{\rho}$ is still a diffeomorphism between the compact set $[-\epsilon, 1+\epsilon] \times S^{1} \times\left[s_{1}, s_{2}\right]$ and its image.

Remark 5.3. We then have an atlas of charts on $T_{\left[s_{1}, s_{2}\right]}$ taking values in $\partial_{\infty} \mathbb{H}_{\mathbb{C}}^{2}$ by choosing lifts of $T_{\left[s_{1}, s_{2}\right]}$ in the space $[-\epsilon, 1+\epsilon] \times S^{1} \times\left[s_{1}, s_{2}\right] \subset \widetilde{T}_{\left[s_{1}, s_{2}\right]}$. Transition maps are given by powers of $[U]=\rho(l)$.

Fix $s_{1}<s_{1}^{\prime}<s_{2}^{\prime}<s_{2}$.

Lemma 5.4 (straightening). If $\rho$ is close enough to $\rho_{0}$, perhaps after taking an isotopy of $\operatorname{Dev}_{\rho}$, we have, for all $(t, \zeta, s) \in \mathbb{R} \times S^{1} \times\left[s_{1}^{\prime}, s_{2}^{\prime}\right]$,

$$
\operatorname{Dev}_{\rho}(t, \zeta, s)=\phi_{t}^{\rho}\left(\operatorname{Dev}_{\rho}(0, \zeta, s)\right)
$$


Proof. The flows $\phi_{t}^{\rho}$ and $\phi_{t}^{\rho_{0}}$ are close in the $\mathcal{C}^{1}$ topology when $\rho$ is close to $\rho_{0}$. We deduce that the deformation from $\rho_{0}$ to $\rho$ induces a $\mathcal{C}^{1}$ deformation from $\phi_{t}^{\rho_{0}} \circ \operatorname{Dev}_{0}$ to $\phi_{t}^{\rho} \circ \operatorname{Dev}_{\rho}$. First we restrict to the compact set $[0,1] \times S^{1} \times\left[s_{1}^{\prime}, s_{2}^{\prime}\right]$, which is in the interior of $[-\epsilon, 1+\epsilon] \times S^{1} \times\left[s_{1}, s_{2}\right]$.

Since

$$
\operatorname{Dev}_{0}\left([0,1] \times S^{1} \times\left[s_{1}^{\prime}, s_{2}^{\prime}\right]\right)=\bigcup_{t \in[0,1]} \phi_{t}^{\rho_{0}}\left(\{0\} \times S^{1} \times\left[s_{1}^{\prime}, s_{2}^{\prime}\right]\right),
$$

if $\rho$ is close enough to $\rho_{0}$,

$$
\bigcup_{t \in[0,1]} \phi_{t}^{\rho}\left(\{0\} \times S^{1} \times\left[s_{1}^{\prime}, s_{2}^{\prime}\right]\right)
$$

is contained in the interior of $\operatorname{Dev}_{\rho}\left([0,1] \times S^{1} \times\left[s_{1}, s_{2}\right]\right)$.

Since $[U] \cdot \phi_{t}^{\rho}=\phi_{t+1}^{\rho}$ and $[U] \cdot \operatorname{Dev}_{\rho}(t, \zeta, s)=\operatorname{Dev}_{\rho}(t+1, \zeta, s)$, we can straighten $\operatorname{Dev}_{\rho}$ by a $[U]$-equivariant isotopy to have, for $(t, \zeta, s) \in \mathbb{R} \times S^{1} \times\left[s_{1}^{\prime}, s_{2}^{\prime}\right]$,

$$
\operatorname{Dev}_{\rho}(t, \zeta, s)=\phi_{t}^{\rho}\left(\operatorname{Dev}_{\rho}(0, \zeta, s)\right)
$$

From now on, we suppose that for all $(t, \zeta, s) \in \mathbb{R} \times S^{1} \times\left[s_{1}^{\prime}, s_{2}^{\prime}\right]$ we have $\operatorname{Dev}_{\rho}(t, \zeta, s)=\phi_{t}^{\rho}\left(\operatorname{Dev}_{\rho}(0, \zeta, s)\right)$.

Lemma 5.5. Let $C$ be a $\mathbb{C}$-circle invariant by $[U]$. Then $C$ and the annulus $\operatorname{Dev}_{\rho}\left(\{0\} \times S^{1} \times\left[s_{1}^{\prime}, s_{2}^{\prime}\right]\right)$ are not linked.

Proof. First, $[U]$ is a regular element close to the unipotent element $\left[U_{0}\right]$, which has fixed point $p_{0} \in \partial_{\infty} \mathbb{H}_{\mathbb{C}}^{2}$. Thanks to Remarks 2.22 and 2.23, we know that $C$ leaves every compact subset of $\partial_{\infty} \mathbb{H}_{\mathbb{C}}^{2}-\left\{p_{0}\right\}$ when $[U]$ approaches $\left[U_{0}\right]$. Since $\operatorname{Dev}_{\rho}\left(\{0\} \times S^{1} \times\left[s_{1}^{\prime}, s_{2}^{\prime}\right]\right)$ stays in a fixed compact set when we deform $\rho_{0}$ to $\rho$, we deduce that $C$ and the annulus $\operatorname{Dev}_{\rho}\left(\{0\} \times S^{1} \times\left[s_{1}^{\prime}, s_{2}^{\prime}\right]\right)$ are not linked.

It only remains to establish a local surgery result, similar to the result contained in [Schwartz 2007, Chapter 8].

Thanks to Lemma 5.4, we know that $\operatorname{Dev}_{\rho}\left(\widetilde{T}_{\left[s_{1}^{\prime}, s_{2}^{\prime}-\epsilon\right]}\right)$ is the orbit by $\phi_{t}^{\rho}$ of the annulus $A=\operatorname{Dev}_{\rho}\left(\{0\} \times S^{1} \times\left[s_{1}^{\prime}, s_{2}^{\prime}-\epsilon\right]\right)$. This orbit separates $\partial_{\infty} \mathbb{H}_{\mathbb{C}}^{2}$ (if $[U]$ is elliptic) or $\partial_{\infty} \mathbb{H}_{\mathbb{C}}^{2}$ minus two points (if $[U]$ is loxodromic) into two connected components $C_{1}$ and $C_{2}$, with respective boundaries $\operatorname{Dev}_{\rho}\left(\widetilde{T}_{s_{1}^{\prime}}\right)$ and $\operatorname{Dev}_{\rho}\left(\widetilde{T}_{s_{2}^{\prime}}\right)$. We have a proper action of $[U]$ on $C_{2}$, and so we can consider the quotient manifold $N=C_{2} /\langle[U]\rangle$. It is a compact manifold with a torus boundary, endowed with a spherical CR structure which coincides with the structure of $M_{\left[0, s_{2}^{\prime}[\right.}$ on $T_{] s_{2}^{\prime}-\epsilon, s_{2}^{\prime}}$. Thus, the gluing $M_{\left[0, s_{2}^{\prime}[\right.} \cup N / \sim$ has a spherical CR structure which extends the structure $\left(\operatorname{Dev}_{\rho}, \rho\right)$ of $M$.

We will show that if $[U]$ is loxodromic or elliptic of type $(p / n, 1 / n)$, then $N$ is a solid torus and that we have a spherical CR structure on a Dehn surgery of $M$ of 


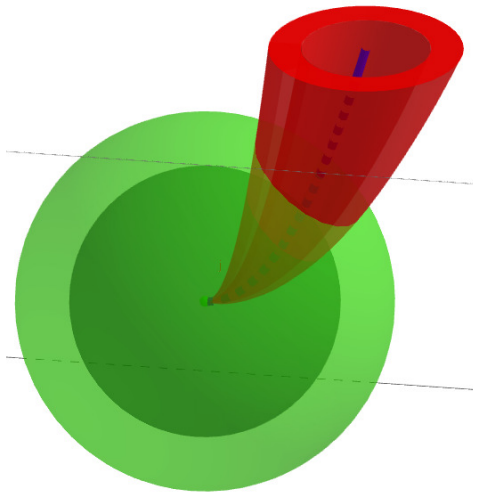

Figure 9. The orbit of $A$ under $\phi_{t}$ (red) and the spheres $S$ and $[U] \cdot S$ (green).

a certain slope. If [ $U$ ] is elliptic of type $(p / n, q / n)$, we will see a description of $N$ as a complement of a torus knot in some lens space.

Case 1: $[U]$ is loxodromic. We work in the Siegel model, and we identify $\partial_{\infty} \mathbb{H}_{\mathbb{C}}^{2}$ with $(\mathbb{C} \times \mathbb{R}) \cup\{\infty\}$. Perhaps after conjugating, we can assume that there exists $\lambda \in \mathbb{C}$ such that $|\lambda|>1$ and

$$
U=T_{\lambda}=\left(\begin{array}{ccc}
\lambda & 0 & 0 \\
0 & \bar{\lambda} / \lambda & 0 \\
0 & 0 & 1 / \bar{\lambda}
\end{array}\right) .
$$

Note that $[U]$ has two fixed points: $(0,0)$ and $\infty$. Let $S$ be the sphere centered at $(0,0)$ and of radius 1 in $\mathbb{C} \times \mathbb{R}$. This sphere is a fundamental domain for the action of the flow $\phi_{t}^{\rho}$. The subgroup generated by $[U]$ acts properly on $(\mathbb{C} \times \mathbb{R})-(0,0)$, and the region $\bigcup_{t \in[0,1]} \phi_{t}^{\rho}(S)$ with boundary components $S$ and $[U] \cdot S$ is a fundamental domain for this action. The orbit of $A$ under $\phi_{t}^{\rho}$ intersects $S$ in an annulus that separates $S$ into two disks $D_{1}$ and $D_{2}$, so that their orbits under $\phi_{t}$ are the connected components $C_{1}$ and $C_{2}$ respectively. Figure 9 shows this situation.

The quotient manifold $N=C_{2} /\langle[U]\rangle$ is obtained by identifying $D_{2}$ and $[U] \cdot D_{2}$ in $\bigcup_{t \in[0,1]} \phi_{t}^{\rho}\left(D_{2}\right)$. Thus, it is a solid torus. But the curve of $\pi_{1}(T)$ that becomes trivial in $C_{2}$ is the one homotopic to the boundary of $D_{2}$ : so it is $m$. We deduce that the surgery is of type $(0,1)$.

Case 2: $[U]$ is elliptic of type $(p / n, \pm 1 / n)$. By choosing $\left[U_{0}\right]^{ \pm 1}$ instead of $\left[U_{0}\right]$ as the generator of the peripheral holonomy, we can suppose that $U$ is of type $( \pm p / n, 1 / n)$. For ease of exposition, we write the proof for $[U]$ of type $(p / n, 1 / n)$.

We reason in the same way as in the loxodromic case. By Lemma 5.5, we know that $\operatorname{Dev}_{\rho}\left(\widetilde{T}_{\left[s_{1}^{\prime}, s_{2}^{\prime}-\epsilon\right]}\right)$ is the orbit under $\phi_{t}$ of the annulus $A=\operatorname{Dev}_{\rho}\left(\{0\} \times S^{1} \times\left[s_{1}^{\prime}, s_{2}^{\prime}\right]\right)$, which is not linked to any of the invariant $\mathbb{C}$-circles of $[U]$. 


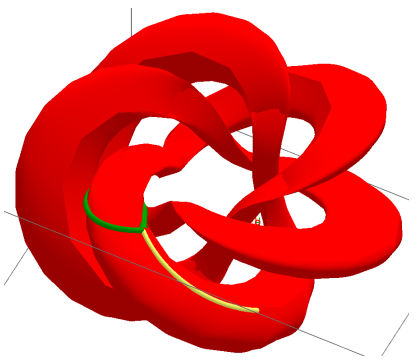

Figure 10. The orbit of $A$ under $\phi_{t}$ (in red), the longitude $l$ (yellow), and the meridian $m$ (green).

The orbit of $A$ under the flow $\phi_{t}^{\rho}$ is then homeomorphic to $S^{1} \times S^{1} \times\left[s_{1}^{\prime}, s_{2}^{\prime}\right]$. Its complement in $\partial_{\infty} \mathbb{H}_{\mathbb{C}}^{2}$ has two connected components; let $C_{2}$ be the component with boundary $\operatorname{Dev}_{\rho}\left(\widetilde{T}_{s_{2}^{\prime}}\right)$. Following Remark 2.15 , the orbits of the flow are not knotted: the two connected components are solid tori, and $[U]$ acts properly on each one. But the quotient of a solid torus by a proper action of a finite group is still a solid torus. The quotient manifold $N=C_{2} /\langle[U]\rangle$ is then a solid torus, and we have a spherical CR structure on a Dehn surgery of $M$. It only remains to identify it.

Perhaps after a conjugation, we can assume that

$$
U=e^{-2 i \pi(p+1) /(3 n)}\left(\begin{array}{ccc}
e^{2 i \pi p / n} & 0 & 0 \\
0 & e^{2 i \pi / n} & 0 \\
0 & 0 & 1
\end{array}\right)
$$

in the ball model. In the Siegel model, by identifying $\partial_{\infty} \mathbb{M}_{\mathbb{C}}^{2}$ with $(\mathbb{C} \times \mathbb{R}) \cup\{\infty\}$, we have that $\left[C U C^{-1}\right]$ stabilizes two $\mathbb{C}$-circles: the circle $\mathcal{C}_{1}$ centered at 0 of radius $\sqrt{2}$ in $\mathbb{C} \times\{0\}$ and $\mathcal{C}_{2}$ the axis $\{0\} \times \mathbb{R}$. A generic orbit of the flow turns once around $\mathcal{C}_{1}$ and $p$ times around $\mathcal{C}_{2}$.

Let $\gamma$ be the loop that follows the $\mathbb{C}$-circle $\mathcal{C}_{2}$ and is oriented so that the meridian $m$ is homotopic to $\gamma$ in the component $C_{2}$. In this case, $n l$ is homotopic, also in $C_{2}$, to $-p \gamma$. Thus $n l+p m$ is a homotopically trivial loop in $C_{2}$, which is a covering of the solid torus $N$ glued to $M$. So it is also a trivial loop in $N$. We deduce that the surgery is of type $(n, p)$.

Case 3: $[U]$ is elliptic of type $(p / n, q / n)$. As in Cases 1 and 2 above, we know that $\operatorname{Dev}_{\rho}\left(\widetilde{T}_{\left[s_{1}^{\prime}, s_{2}^{\prime}-\epsilon\right]}\right)$ is the orbit by $\phi_{t}$ of the annulus $A=\operatorname{Dev}_{\rho}\left(\{0\} \times S^{1} \times\left[s_{1}^{\prime}, s_{2}^{\prime}\right]\right)$, which is not linked to any of the invariant $\mathbb{C}$-circles of $[U]$.

The orbit of $A$ under the flow $\phi_{t}^{\rho}$ is homeomorphic to $S^{1} \times S^{1} \times\left[s_{1}^{\prime}, s_{2}^{\prime}\right]$. Its complement in $\partial_{\infty} \mathbb{H}_{\mathbb{C}}^{2}$ has two connected components. Let $C_{2}$ be (again) the component with boundary $\operatorname{Dev}_{\rho}\left(\widetilde{T}_{s_{2}^{\prime}}\right)$ and $C_{1}$ the one with boundary $\operatorname{Dev}_{\rho}\left(\widetilde{T}_{s_{1}^{\prime}}\right)$. According to Remark 2.15, generic orbits of the flow are torus knots of type $(p, q)$ : $C_{1}$ is then a tubular neighborhood of one of the orbits and $C_{2}$ is homeomorphic to 
the complement of a torus knot of type $(p, q)$. But $[U]$ acts properly on $\partial_{\infty} \mathbb{M}_{\mathbb{C}}^{2}$ and stabilizes $C_{1}$ and $C_{2}$.

Notice that, in the ball model, the action of the group generated by $[U]$ is the same as the one of the group generated by

$$
\left(z_{1}, z_{2}\right) \mapsto\left(e^{2 i \pi / n} z_{1}, e^{2 i \pi \alpha / n} z_{2}\right),
$$

where $\alpha \equiv p^{-1} q \bmod n$. The quotient $\partial_{\infty} \mathbb{H}_{\mathbb{C}}^{2} /\langle[U]\rangle$ is then homeomorphic to the lens space $L(n, \alpha)$. Furthermore, $C_{1} /\langle[U]\rangle$ is a solid torus knotted in $\partial_{\infty} \mathbb{H}_{\mathbb{C}}^{2} /\langle[U]\rangle$. The quotient manifold $V(p, q, n)=C_{2} /\langle[U]\rangle$ is the complement of a torus knot in $\partial_{\infty} \mathbb{H}_{\mathbb{C}}^{2} /\langle[U]\rangle \simeq L(n, \alpha)$. The spherical CR structure of $M$ then extends to the gluing of $M$ and $V(p, q, n)$ along their torus boundary components.

\section{Acknowledgements}

The author thanks his advisors Martin Deraux and Antonin Guilloux, as well as Elisha Falbel and the referee for the many discussions and useful remarks on this paper.

\section{References}

[Bergeron and Gelander 2004] N. Bergeron and T. Gelander, "A note on local rigidity", Geom. Dedicata 107 (2004), 111-131. MR 2110758 Zbl 1062.22028

[Cooper et al. 2000] D. Cooper, C. D. Hodgson, and S. P. Kerckhoff, Three-dimensional orbifolds and cone-manifolds, MSJ Memoirs 5, Mathematical Society of Japan, Tokyo, 2000. MR 1778789 Zbl 0955.57014

[Deraux 2014] M. Deraux, "A 1-parameter family of spherical CR uniformizations of the figure eight knot complement", preprint, 2014. To appear in Geom. Topol. arXiv 1410.1198

[Deraux 2015] M. Deraux, "On spherical CR uniformization of 3-manifolds", Exp. Math. 24:3 (2015), 355-370. MR 3359222

[Deraux and Falbel 2015] M. Deraux and E. Falbel, "Complex hyperbolic geometry of the figure eight knot”, Geometry \& Topology 19:1 (2015), 237-293. Zbl 1335.32028

[Falbel et al. 2016] E. Falbel, A. Guilloux, P.-V. Koseleff, F. Rouillier, and M. Thistlethwaite, "Character varieties for SL(3, $\mathbb{C})$ : the figure eight knot”, Exp. Math. 25:2 (2016), 219-235. MR 3463570

[Genzmer 2010] J. Genzmer, Sur les triangulations des structures CR-sphériques, Ph.D. thesis, Université Pierre et Marie Curie, 2010, available at https://tel.archives-ouvertes.fr/tel-00502363/ document.

[Goldman 1999] W. M. Goldman, Complex hyperbolic geometry, Clarendon, New York, 1999. MR 1695450 Zbl 0939.32024

[Goldman 2010] W. M. Goldman, "Locally homogeneous geometric manifolds", pp. 717-744 in Proceedings of the International Congress of Mathematicians, II, edited by R. Bhatia et al., Hindustan Book Agency, New Delhi, 2010. MR 2827816 Zbl 1234.57001

[Parker et al. 2016] J. R. Parker, J. Wang, and B. Xie, "Complex hyperbolic $(3,3, n)$ triangle groups", Pacific J. Math. 280:2 (2016), 433-453. MR 3453571 Zbl 06537059 
[Schwartz 2007] R. E. Schwartz, Spherical CR geometry and Dehn surgery, Annals of Mathematics Studies 165, Princeton University Press, 2007. MR 2286868 Zbl 1116.57016

[Thurston 2002] W. P. Thurston, “The geometry and topology of three-manifolds", lecture notes, Princeton Univ., 2002, available at http://www.msri.org/gt3m/.

Received September 14, 2015. Revised January 21, 2016.

Miguel ACOSTA

UMR 7586 CNRS

Université Pierre et Marie Curie

4 Place Jussieu

75252 PARIS CEDEX 05

FRANCE

miguel.acosta@imj-prg.fr 


\title{
PACIFIC JOURNAL OF MATHEMATICS
}

Founded in 1951 by E. F. Beckenbach (1906-1982) and F. Wolf (1904-1989)

$$
\text { msp.org/pjm }
$$

\section{EDITORS}

\author{
Don Blasius (Managing Editor) \\ Department of Mathematics \\ University of California \\ Los Angeles, CA 90095-1555 \\ blasius@math.ucla.edu
}

\author{
Paul Balmer \\ Department of Mathematics \\ University of California \\ Los Angeles, CA 90095-1555 \\ balmer@math.ucla.edu \\ Robert Finn \\ Department of Mathematics \\ Stanford University \\ Stanford, CA 94305-2125 \\ finn@math.stanford.edu \\ Sorin Popa \\ Department of Mathematics \\ University of California \\ Los Angeles, CA 90095-1555 \\ popa@math.ucla.edu
}

\author{
Vyjayanthi Chari \\ Department of Mathematics \\ University of California \\ Riverside, CA 92521-0135 \\ chari@math.ucr.edu \\ Kefeng Liu \\ Department of Mathematics \\ University of California \\ Los Angeles, CA 90095-1555 \\ liu@math.ucla.edu \\ Igor Pak \\ Department of Mathematics \\ University of California \\ Los Angeles, CA 90095-1555 \\ pak.pjm@gmail.com \\ Paul Yang \\ Department of Mathematics \\ Princeton University \\ Princeton NJ 08544-1000 \\ yang@math.princeton.edu
}

\section{PRODUCTION}

Silvio Levy, Scientific Editor, production@msp.org

\section{SUPPORTING INSTITUTIONS}

ACADEMIA SINICA, TAIPEI

CALIFORNIA INST. OF TECHNOLOGY

STANFORD UNIVERSITY

UNIV. OF BRITISH COLUMBIA

UNIV. OF CALIFORNIA, BERKELEY

UNIV. OF CALIFORNIA, DAVIS

UNIV. OF CALIFORNIA, LOS ANGELES

UNIV. OF CALIFORNIA, RIVERSIDE

UNIV. OF CALIFORNIA, SAN DIEGO

UNIV. OF CALIF., SANTA BARBARA
KEIO UNIVERSITY

MATH. SCIENCES RESEARCH INSTITUTE

NEW MEXICO STATE UNIV.

OREGON STATE UNIV.
Daryl Cooper

Department of Mathematics

University of California

Santa Barbara, CA 93106-3080 cooper@math.ucsb.edu

Jiang-Hua Lu

Department of Mathematics

The University of Hong Kong

Pokfulam Rd., Hong Kong

jhlu@maths.hku.hk

$$
\text { Jie Qing }
$$

Department of Mathematics

University of California

Santa Cruz, CA 95064

qing@ cats.ucsc.edu

\author{
UNIV. OF CALIF., SANTA CRUZ \\ UNIV. OF MONTANA \\ UNIV. OF OREGON \\ UNIV. OF SOUTHERN CALIFORNIA \\ UNIV. OF UTAH \\ UNIV. OF WASHINGTON \\ WASHINGTON STATE UNIVERSITY
}

These supporting institutions contribute to the cost of publication of this Journal, but they are not owners or publishers and have no responsibility for its contents or policies.

See inside back cover or msp.org/pjm for submission instructions.

The subscription price for 2016 is US $\$ 440 /$ year for the electronic version, and \$600/year for print and electronic.

Subscriptions, requests for back issues and changes of subscriber address should be sent to Pacific Journal of Mathematics, P.O. Box 4163, Berkeley, CA 94704-0163, U.S.A. The Pacific Journal of Mathematics is indexed by Mathematical Reviews, Zentralblatt MATH, PASCAL CNRS Index, Referativnyi Zhurnal, Current Mathematical Publications and Web of Knowledge (Science Citation Index).

The Pacific Journal of Mathematics (ISSN 0030-8730) at the University of California, c/o Department of Mathematics, 798 Evans Hall \#3840, Berkeley, CA 94720-3840, is published twelve times a year. Periodical rate postage paid at Berkeley, CA 94704, and additional mailing offices. POSTMASTER: send address changes to Pacific Journal of Mathematics, P.O. Box 4163, Berkeley, CA 94704-0163.

PJM peer review and production are managed by EditFLOW ${ }^{\circledR}$ from Mathematical Sciences Publishers.

PUBLISHED BY

\section{I. mathematical sciences publishers}

nonprofit scientific publishing

http://msp.org/

(C) 2016 Mathematical Sciences Publishers 


\section{PACIFIC JOURNAL OF MATHEMATICS}

Volume $284 \quad$ No. $2 \quad$ October 2016

Spherical CR Dehn surgeries

Miguel ACOsta

Degenerate flag varieties and Schubert varieties: a characteristic free approach

283

Giovanni Cerulli Irelli, Martina LANini and Peter

LITTELMANN

Solitons for the inverse mean curvature flow

Gregory Drugan, HoJoo LEE and GLEN WHEELER

Bergman theory of certain generalized Hartogs triangles

\section{LUKE D. EDHOLM}

Transference of certain maximal Hilbert transforms on the torus

DASHAN FAN, HUOXIONG WU and FAYOU ZHAO

The Turaev and Thurston norms

STEFAN FriedL, DANIEL S. Silver and Susan G. WiLLiams

A note on nonunital absorbing extensions

JAMES GABE

On nonradial singular solutions of supercritical biharmonic equations

Zongming GuO, JUNCHENG WeI and Wen YANG

Natural commuting of vanishing cycles and the Verdier dual

\section{DAVID B. MASSEY}

The nef cones of and minimal-degree curves in the Hilbert schemes of points on certain surfaces

ZHENBo QIN and YUPING TU

Smooth approximation of conic Kähler metric with lower Ricci curvature bound

\section{LIANGMING SHEN}

Maps from the enveloping algebra of the positive Witt algebra to regular algebras

Susan J. SierRa and Chelsea Walton 